\title{
DEGREE CONES AND MONOMIAL BASES OF LIE ALGEBRAS AND QUANTUM GROUPS
}

\author{
TEODOR BACKHAUS and XIN FANG \\ Mathematisches Institut, Universität zu Köln, Cologne, North Rhine-Westphalia, Germany \\ e-mails: tbackha@math.uni-koeln.de,xinfang.math@gmail.com
}

and GHISLAIN FOURIER

School of Mathematics and Statistics, University of Glasgow, United Kingdom Institut für Algebra, Zahlentheorie und Diskrete Mathematik, Leibniz Universität Hannover, Hannover, Lower Saxony, Germany e-mail:fourier@math.uni-hannover.de

(Received 10 June 2016; revised 29 September 2016; accepted 30 October 2016; first published online 20 March 2017)

\begin{abstract}
We provide $\mathbb{N}$-filtrations on the negative part $U_{q}\left(\mathfrak{n}^{-}\right)$of the quantum group associated to a finite-dimensional simple Lie algebra $\mathfrak{g}$, such that the associated graded algebra is a skew-polynomial algebra on $\mathfrak{n}^{-}$. The filtration is obtained by assigning degrees to Lusztig's quantum PBW root vectors. The possible degrees can be described as lattice points in certain polyhedral cones. In the classical limit, such a degree induces an $\mathbb{N}$-filtration on any finite-dimensional simple $\mathfrak{g}$-module. We prove for type $A_{n}, C_{n}, B_{3}, D_{4}$ and $G_{2}$ that a degree can be chosen such that the associated graded modules are defined by monomial ideals, and conjecture that this is true for any $\mathfrak{g}$.
\end{abstract}

\section{Introduction.}

1.1. Motivation. Let $\mathfrak{g}$ be a finite-dimensional simple Lie algebra with a triangular decomposition $\mathfrak{g}=\mathfrak{n}^{+} \oplus \mathfrak{h} \oplus \mathfrak{n}^{-}$. Let $U\left(\mathfrak{n}^{-}\right)$be the corresponding universal enveloping algebra. Lusztig [18] introduced the canonical basis for the quantized enveloping algebra $U_{q}\left(\mathfrak{n}^{-}\right)$. Subsequently, Kashiwara [14] gave a different construction of this basis under the name global crystal basis. When the quantum parameter is specialized to 1 , the canonical basis is specialized to a linear basis $\mathcal{B}$ of $U\left(\mathfrak{n}^{-}\right)$. Let $V(\lambda)$ be the finite-dimensional irreducible representation of $U(\mathfrak{g})$ of highest weight $\lambda$ and $v_{\lambda}$ be a fixed highest weight vector. The canonical basis $\mathcal{B}$ of $U\left(\mathfrak{n}^{-}\right)$induces a canonical basis of $V(\lambda)$ by

$$
\mathcal{B}_{\lambda}=\left\{b \cdot v_{\lambda} \mid b \in \mathcal{B}, b \cdot v_{\lambda} \neq 0\right\}
$$

This is one of the most important properties of the canonical basis.

In this paper, motivated by [7-9], we are interested in the existence of monomial bases $\mathcal{E}$ of $U\left(\mathfrak{n}^{-}\right)$satisfying the following properties:

(P1) There exists an $\mathbb{N}$-filtration $\mathcal{F}$ on $U\left(\mathfrak{n}^{-}\right)$such that the associated graded algebra is the polynomial algebra $S\left(\mathfrak{n}^{-}\right)$; the set $\mathcal{E}$ is a linear basis of the associated graded algebra. 
(P2) Let $V^{\mathcal{F}}(\lambda)$ be the associated graded $S\left(\mathfrak{n}^{-}\right)$-module with cyclic vector $v_{\lambda}^{\mathcal{F}}$,

$$
\mathcal{E}_{\lambda}=\left\{b \cdot v_{\lambda}^{\mathcal{F}} \mid b \in \mathcal{E}, b \cdot v_{\lambda}^{\mathcal{F}} \neq 0\right\}
$$

is a linear basis of $V^{\mathcal{F}}(\lambda)$, hence a linear basis of $V(\lambda)$.

An equivalent formulation of $(\mathrm{P} 2)$ in terms of monomial ideals is:

(P2') find an $\mathbb{N}$-filtration $\mathcal{F}$ on $U\left(\mathfrak{n}^{-}\right)$such that for any dominant integral weight $\lambda$, the defining ideal of $\operatorname{gr}^{\mathcal{F}} V(\lambda)$ is monomial.

By turning back to quantum groups, we may ask similar questions:

(Q1) Is there an $\mathbb{N}$-filtration of $U_{q}\left(\mathfrak{n}^{-}\right)$such that the associated graded algebra is $S_{q}\left(\mathfrak{n}^{-}\right)$ (a skew-polynomial algebra on the vector space $\mathfrak{n}^{-}$).

1.2. Answering (Q1). The answer to (P1) is rather trivial. We fix a weighted basis of $\mathfrak{n}^{-}$, indexed by positive roots $\Delta^{+}$, and let $\mathbf{d}: \mathfrak{n}^{-} \longrightarrow \mathbb{N}$ be a degree function on $\mathfrak{n}^{-}$ such that for any basis elements $x, y \in \mathfrak{n}^{-}$:

$$
\mathbf{d}(x)+\mathbf{d}(y)>\mathbf{d}([x, y]) .
$$

This induces an $\mathbb{N}$-filtration on $U\left(\mathfrak{n}^{-}\right)$and the induced associated graded algebra is isomorphic to $S\left(\mathfrak{n}^{-}\right)$. We denote $\mathcal{D}$, called the classical degree cone, the real cone generated by all degree functions on $\mathfrak{n}^{-}$(respectively $\Delta^{+}$) satisfying these inequalities.

To construct an $\mathbb{N}$-filtration on $U_{q}\left(\mathfrak{n}^{-}\right)$, it is not enough to consider its Chevalley generators $F_{1}, \ldots, F_{n}$, since $U_{q}\left(\mathfrak{n}^{-}\right)$is already a graded algebra for any grading on these generators, and the defining ideals of simple modules are seldom monomial.

There is another basis of $U_{q}\left(\mathfrak{n}^{-}\right)$given by Lusztig [19], called quantum PBW basis. Let $\underline{w}_{0}=s_{i_{1}} \ldots s_{i_{N}}$ be a reduced decomposition of the longest Weyl group element. We associate a sequence of elements $F_{\beta_{1}}, \ldots, F_{\beta_{N}} \in U_{q}\left(\mathfrak{n}^{-}\right)$, where $\left\{\beta_{1}, \ldots, \beta_{N}\right\}$ is the set of positive roots and $F_{\beta_{i}}$ is a quantum PBW root vector of weight $-\beta_{i}$. Then, Lusztig has shown that ordered monomials in $F_{\beta_{1}}, \ldots, F_{\beta_{N}}$ form a linear basis of $U_{q}\left(\mathfrak{n}^{-}\right)$.

The naive approach of setting the degree of $F_{\beta_{i}}$ to 1 for all $\beta_{i}$ does not provide $\operatorname{gr} U_{q}\left(\mathfrak{n}^{-}\right) \cong S_{q}\left(\mathfrak{n}^{-}\right)$for the induced filtration:

EXAMPLE. Let $\mathfrak{g}=\mathfrak{s l}_{4}$ be of type $\mathrm{A}_{3}$. Fix the reduced decomposition $\underline{w}_{0}=s_{1} s_{2} s_{1} s_{3} s_{2} s_{1}$ of the longest element $w_{0}$ in the Weyl group of $\mathfrak{g}$. We denote by $F_{i i+1 \ldots k}$ the quantum PBW root vector corresponding to the root $-\left(\alpha_{i}+\alpha_{i+1}+\cdots+\alpha_{k}\right)$. The following relation holds in $U_{q}\left(\mathfrak{n}^{-}\right)$:

$$
F_{23} F_{12}=F_{12} F_{23}-\left(q-q^{-1}\right) F_{2} F_{123} .
$$

In general, the commutation relations in $U_{q}\left(\mathfrak{n}^{-}\right)$are given by the following Levendorskii-Soibelman ( $\mathrm{L}-\mathrm{S}$ for short) formula: for any $i<j$ :

$$
F_{\beta_{j}} F_{\beta_{i}}-q^{-\left(\beta_{i}, \beta_{j}\right)} F_{\beta_{i}} F_{\beta_{j}}=\sum_{n_{i+1}, \ldots, n_{j-1} \geq 0} c\left(n_{i+1}, \ldots, n_{j-1}\right) F_{\beta_{i+1}}^{n_{i+1}} \ldots F_{\beta_{j-1}}^{n_{j-1}} .
$$

These commutation relations depend heavily on the choice of reduced decomposition $\underline{w}_{0}$. For a given reduced decomposition $\underline{w}_{0}$, we seek for degree functions on the set of 
positive roots

$$
\mathbf{d}: \Delta_{+} \longrightarrow \mathbb{N}
$$

such that letting $\operatorname{deg}\left(F_{\beta}\right)=\mathbf{d}(\beta)$ for $\beta \in \Delta_{+}$defines a filtered algebra structure on $U_{q}\left(\mathfrak{n}^{-}\right)$and the associated graded algebra satisfies $\operatorname{gr}^{\mathbf{d}} U_{q}\left(\mathfrak{n}^{-}\right) \cong S_{q}\left(\mathfrak{n}^{-}\right)$. Inspired by the $\mathrm{L}-\mathrm{S}$ formula, we define for any reduced decomposition $\underline{w}_{0}$ the quantum degree cone $\mathcal{D}_{\underline{w}_{0}}^{q}$ by

$$
\mathcal{D}_{\underline{w}_{0}}^{q}:=\left\{\left(d_{\beta}\right) \in \mathbb{R}_{+}^{\left|\Delta_{+}\right|} \mid \text {for any } i<j, d_{\beta_{i}}+d_{\beta_{j}}>\sum_{k=i+1}^{j-1} n_{k} d_{\beta_{k}} \text { if } c\left(n_{i+1}, \ldots, n_{j-1}\right) \neq 0\right\} \text {. }
$$

The first main theorem of this paper is:

TheOrem A. Let $\underline{w}_{0}$ be a reduced decomposition. Then,

(1) the set $\mathcal{D}_{\underline{w}_{0}}^{q}$ is a non-empty, open polyhedral cone;

(2) a degree function $\mathbf{d}: \Delta^{+} \longrightarrow \mathbb{N}$ defines a filtered algebra structure on $U_{q}\left(\mathfrak{n}^{-}\right)$ such that $\operatorname{gr}^{\mathbf{d}} U_{q}\left(\mathfrak{n}^{-}\right) \cong S_{q}\left(\mathfrak{n}^{-}\right)$if and only if $\mathbf{d} \in \mathcal{D}_{\underline{w}_{0}}^{q} \cap \mathbb{Z}^{N}$.

It is natural to ask whether there is a uniform degree function $\mathbf{d}$ which is compatible with every reduced decomposition. We will show that for a simple Lie algebra $\mathfrak{g}$, such a function exists if and only if the rank of $\mathfrak{g}$ is less or equal than 2, i.e. for any $\mathfrak{g}$ of rank larger than 2, we have

$$
\bigcap_{\underline{w}_{0} \in R\left(w_{0}\right)} \mathcal{D}_{\underline{w}_{0}}^{q}=\emptyset
$$

where $R\left(w_{0}\right)$ is the set of all reduced decompositions of $w_{0}$.

Suppose $\mathfrak{g}$ is of simply laced type and the reduced decomposition is adapted to an orientation of the associated Dynkin quiver. Using the Hall algebra realization of $U_{q}\left(\mathfrak{n}^{-}\right)$, the coordinates of the lattice points in the quantum degree cone have an interpretation as dimensions of certain homomorphism spaces for the particular Dynkin quiver [7].

1.3. Answering (P2'). We turn from the quantum situation to the classical one and analyse the implication of the induced filtration for finite-dimensional simple modules.

Let $V(\lambda)$ be the simple module of highest weight $\lambda$. Since $V(\lambda)=U\left(\mathfrak{n}^{-}\right) \cdot v_{\lambda}$, any filtration on $U\left(\mathfrak{n}^{-}\right)$induces a filtration on $V(\lambda)$.

Let $\mathbf{d}: \Delta_{+} \rightarrow \mathbb{N}$ be a degree function for $U\left(\mathfrak{n}^{-}\right)$such that $\operatorname{gr}^{\mathbf{d}} U\left(\mathfrak{n}^{-}\right) \cong S\left(\mathfrak{n}^{-}\right)$. The associated graded module $\operatorname{gr}^{\mathbf{d}} V(\lambda)$ of the induced filtration is a cyclic $S\left(\mathfrak{n}^{-}\right)$-module. Hence, there exists an ideal $I^{\mathbf{d}}(\lambda) \subset S\left(\mathfrak{n}^{-}\right)$such that $\operatorname{gr}^{\mathbf{d}} V(\lambda) \cong S\left(\mathfrak{n}^{-}\right) / I^{\mathbf{d}}(\lambda)$.

Our second aim of the paper is to find monomial bases of $\operatorname{gr}^{\mathbf{d}} V(\lambda)$. If the ideal $I^{\mathbf{d}}(\lambda)$ is monomial, there exists a unique monomial basis for $\operatorname{gr}^{\mathbf{d}} V(\lambda)$. We will focus on this case in the paper.

The global monomial set $\mathcal{S}_{\mathrm{gm}}$ consists of all degree functions $\mathbf{d}: \Delta_{+} \rightarrow \mathbb{N}$ such that for any dominant integral weight $\lambda, I^{\mathrm{d}}(\lambda)$ is a monomial ideal. As the other main result of this paper, all monomial bases appearing in the context of PBW filtration in the literature can be actually obtained through a degree in the global monomial set. 
THEOREM B. Let $\mathfrak{g}$ be a simple Lie algebra of type $\mathrm{A}_{\mathrm{n}}, \mathrm{C}_{\mathrm{n}}, \mathrm{B}_{3}, \mathrm{D}_{4}$ or $\mathrm{G}_{2}$. Then, $\mathcal{S}_{\mathrm{gm}} \neq \emptyset$.

We provide a degree function in the global monomial set in each case (for the $A_{n}$-case this has been done already in [7]). Based on the evidence of several further examples, we conjecture:

Conjecture. (1) $\mathcal{S}_{\mathrm{gm}} \neq \emptyset$ for any simple finite-dimensional Lie algebra $\mathfrak{g}$.

(2) For any simply laced simple Lie algebra, there exists $\underline{w}_{0}$ such that $\mathcal{S}_{\mathrm{gm}} \cap \mathcal{D}_{\underline{w}_{0}}^{q}$ is non-empty.

1.4. Remarks on the boundary of the classical degree cone. Let $\mathfrak{g}$ be of type $A_{n}$. The boundary of $\mathcal{D}$, denoted by $\partial \mathcal{D}$, is defined as the difference of the closure of $\mathcal{D}$ and its relative interior. Let $S(\partial \mathcal{D}):=\partial \mathcal{D} \cap \mathbb{Z}^{\Delta_{+}}$be the lattice points in $\partial \mathcal{D}$.

Let $\mathbf{d} \in S(\partial \mathcal{D})$. Then, $\mathbf{d}$ defines a filtration $\mathcal{F}^{\mathbf{d}}$ on $U\left(\mathfrak{n}^{-}\right)$. In general, the associated graded algebra is no longer the commutative algebra $S\left(\mathfrak{n}^{-}\right)$, but some algebra which is a degenerated version of $U\left(\mathfrak{n}^{-}\right)$and admits a further degeneration to $S\left(\mathfrak{n}^{-}\right)$. This associated graded algebra is the universal enveloping algebra of the Lie algebra $\mathfrak{n}^{-, \mathbf{d}}$, which is a contraction the Lie bracket of $\mathfrak{n}^{-}$on the prescribed roots by $\mathbf{d}$ (see [4] for details).

For $\lambda \in \mathcal{P}_{+}$, we can similarly define the associated graded module $V^{\mathbf{d}}(\lambda)$ : It is a cyclic $U\left(\mathfrak{n}^{-, \mathbf{d}}\right)$-module with cyclic vector $v_{\lambda}^{\mathbf{d}}$. It is proved in [4] that the highest weight orbit

$$
\mathcal{F}^{\mathbf{d}}(\lambda):=\overline{\exp \left(\mathfrak{n}^{-, \mathbf{d}}\right) \cdot\left[v_{\lambda}^{\mathbf{d}}\right]} \subset \mathbb{P}\left(V^{\mathbf{d}}(\lambda)\right)
$$

is a flat degeneration of the partial flag variety $\mathcal{F}(\lambda)$.

Moreover, for some $\mathbf{d} \in S(\partial \mathcal{D})$, it is conjectured in [5] that a monomial basis of the representation $V^{\mathrm{d}}(\lambda)$ can be parameterized by the lattice points in a chain-order polytope associated to a marked poset.

1.5. Organization of paper. In Section 2, we fix notations, introduce the classical degree cone, the local and global monomial sets. Quantum degree cones are defined in Section 3, where Theorem A is proved. We provide examples and properties of the quantum degree cone in Section 4. In Section 5, examples for local and global monomials sets are given and Theorem B is proved. We conclude with some examples on quantum degree cones in Section 6.

\section{Lie algebras and the classical degree cones.}

2.1. Notations and basic properties. Let $\mathfrak{g}$ be a simple Lie algebra of rank $n$ over the field of complex numbers $\mathbb{C}$. We fix a triangular decomposition $\mathfrak{g}=\mathfrak{n}^{+} \oplus \mathfrak{h} \oplus \mathfrak{n}^{-}$ and a set of simple roots $\Pi=\left\{\alpha_{1}, \ldots, \alpha_{n}\right\}$ of $\mathfrak{g}$. The set of positive roots of $\mathfrak{g}$ will be denoted by $\Delta_{+}$with cardinality $N$. Let $\mathcal{Q}_{+}=\sum_{i=1}^{n} \mathbb{N} \varpi_{i}$ be the root monoid. Let $\rho=\frac{1}{2} \sum_{\alpha \in \Delta_{+}} \alpha$ be the half sum of positive roots. For $\alpha \in \Delta_{+}$, we pick a root vector $f_{\alpha}$ of weight $-\alpha$. Let $\varpi_{i}, i=1, \ldots, n$ be the fundamental weights, $\mathcal{P}$ be the weight lattice and $\mathcal{P}_{+}=\sum_{i=1}^{n} \mathbb{N} \varpi_{i}$ be the set of dominant integral weights. For a dominant integral weight $\lambda \in \mathcal{P}_{+}$, let $V(\lambda)$ be the finite-dimensional irreducible representation of $\mathfrak{g}$ of highest weight $\lambda$ and $v_{\lambda}$ a highest weight vector. Let $U\left(\mathfrak{n}^{-}\right)$be the enveloping algebra 
of $\mathfrak{n}^{-}$and $S\left(\mathfrak{n}^{-}\right)$be the symmetric algebra of $\mathfrak{n}^{-}$. For $\lambda=\sum_{i=1}^{n} \lambda_{i} \varpi_{i} \in \mathcal{P}_{+}$, denote the height of $\lambda$ by $|\lambda|:=\sum_{i=1}^{n} m_{i}$.

We define $\mathbb{R}^{\Delta_{+}}:=\left\{f: \Delta_{+} \rightarrow \mathbb{R}\right.$ is a function $\}$. It is an $\mathbb{R}$-vector space of dimension $N$. Let $\mathbb{R}_{\geq 0}^{\Delta_{+}} \subset \mathbb{R}^{\Delta_{+}}$be the set of functions taking positive values, we define similarly $\mathbb{N}^{\Delta_{+}}$and $\mathbb{Z}^{\Delta_{+}}$. A function $\mathbf{d} \in \mathbb{R}^{\Delta_{+}}$is determined by its values $\left(d_{\beta}:=\mathbf{d}(\beta)\right)_{\beta \in \Delta_{+}}$. Once a sequence of positive roots $\left(\beta_{1}, \beta_{2}, \ldots, \beta_{N}\right)$ is fixed, $\mathbb{R}^{\Delta_{+}}$is identified with $\mathbb{R}^{N}$ via: $\mathbf{d} \mapsto\left(d_{\beta_{1}}, d_{\beta_{2}}, \ldots, d_{\beta_{N}}\right)$.

Let $W$ be the Weyl group of $\mathfrak{g}$ with generators $s_{1}, \ldots, s_{n}$ and $w_{0} \in W$ be the longest element. We denote $R\left(w_{0}\right)$ the set of all reduced decompositions of $w_{0}$.

For any reduced decomposition $\underline{w}_{0}=s_{i_{1}} \ldots s_{i_{N}} \in R\left(w_{0}\right)$, we associate a convex total order on $\Delta_{+}$: for $1 \leq t \leq N$, we denote $\beta_{t}=s_{i_{1}} \ldots s_{i_{t-1}}\left(\alpha_{i_{t}}\right)$, then $\Delta_{+}=$ $\left\{\beta_{1}, \ldots, \beta_{N}\right\}$ and $\beta_{1}<\beta_{2}<\ldots<\beta_{N}$ is the desired convex total order. It is proved by Papi [20] that the above association induces a bijection between $R\left(w_{0}\right)$ and the set of all convex total orders on $\Delta_{+}$.

For the simple Lie algebra $\mathfrak{s l}_{n+1}$ of type $\mathrm{A}_{\mathrm{n}}$ and $1 \leq i \leq j \leq n$, we denote $\alpha_{i, j}:=\alpha_{i}+$ $\cdots+\alpha_{j}$, then $\Delta_{+}=\left\{\alpha_{i, j} \mid 1 \leq i \leq j \leq n\right\}$. For the simple Lie algebra of type $\mathrm{B}_{\mathrm{n}}$ and $1 \leq$ $i \leq j \leq n$, we denote $\alpha_{i, j}:=\alpha_{i}+\cdots+\alpha_{j}$ and for $\alpha_{i, j}:=\alpha_{i}+\cdots+\alpha_{n}+\alpha_{n}+\cdots+\alpha_{j}$, then $\Delta_{+}=\left\{\alpha_{i, j}, \alpha_{k, \bar{l}} \mid 1 \leq i \leq j \leq n, 1 \leq k<l \leq n\right\}$. For the simple Lie algebra $\mathfrak{s p}_{2 n}$ of type $\mathrm{C}_{\mathrm{n}}$ and $1 \leq i \leq j \leq n$, we denote $\alpha_{i, j}:=\alpha_{i}+\cdots+\alpha_{j}$ and $\alpha_{i, j}:=\alpha_{i}+\cdots+\alpha_{n}+$ $\cdots+\alpha_{j}$, notice that $\alpha_{i, n}=\alpha_{i, \bar{n}}$, then $\Delta_{+}=\left\{\alpha_{i, j}, \alpha_{i, \bar{j}} \mid 1 \leq i \leq j \leq n\right\}$.

For $\mathbf{s}=\left(s_{\alpha}\right)_{\alpha \in \Delta_{+}} \in \mathbb{N}^{\Delta_{+}}$, we denote $f^{\mathbf{s}}:=\prod_{\alpha \in \Delta_{+}} f_{\alpha}^{s_{\alpha}} \in S\left(\mathfrak{n}^{-}\right)$. For any $\mathbf{d} \in \mathbb{N}^{\Delta_{+}}$, we denote $\operatorname{deg}_{\mathbf{d}}\left(f^{\mathbf{s}}\right):=\sum_{\alpha \in \Delta_{+}} s_{\alpha} d_{\alpha}$.

2.2. The classical degree cone. We start with the classical degree cone.

Definition 2.1. The classical degree cone $\mathcal{D}$ is defined by

$$
\mathcal{D}:=\left\{\mathbf{d} \in \mathbb{R}_{\geq 0}^{\Delta_{+}} \mid \text {for any } \alpha, \beta, \gamma \in \Delta_{+} \text {such that } \alpha+\beta=\gamma, d_{\alpha}+d_{\beta}>d_{\gamma}\right\} .
$$

EXAMPLE 2.2. The element e defined by $e_{\alpha}=1$ for all $\alpha$ is in $\mathcal{D}$ for any simple Lie algebra.

By definition, $\mathcal{D}$ is an open polyhedral cone. We let $S(\mathcal{D}):=\mathcal{D} \cap \mathbb{Z}^{\Delta_{+}}$denote the set of lattice points in $\mathcal{D}$. For any $\mathbf{d}=\left(d_{\beta}\right)_{\beta \in \Delta_{+}} \in S(\mathcal{D})$, we define a filtration $\mathcal{F}^{\mathbf{d}}$ on $U\left(\mathfrak{n}^{-}\right)$by

$$
\mathcal{F}_{s}^{\mathbf{d}} U\left(\mathfrak{n}^{-}\right):=\operatorname{span}\left\{f_{\gamma_{1}} f_{\gamma_{2}} \ldots f_{\gamma_{k}} \mid \gamma_{1}, \ldots, \gamma_{k} \in \Delta_{+} \text {such that } d_{\gamma_{1}}+d_{\gamma_{2}}+\cdots+d_{\gamma_{k}} \leq s\right\} .
$$

By the cyclicity, every irreducible representation $V(\lambda)$ admits a filtration arising from $\mathcal{F}^{\text {d: }}$

$$
\mathcal{F}_{s}^{\mathbf{d}} V(\lambda):=\mathcal{F}_{s}^{\mathbf{d}} U\left(\mathfrak{n}^{-}\right) \cdot v_{\lambda}
$$

Note that for $\mathbf{d}=\mathbf{e}$, this is the PBW filtration, which has been subject to a lot of researches in the past 10 years.

LEMMA 2.3. For any $\mathbf{d} \in S(\mathcal{D})$, we have the following:

(1) $\mathcal{F}^{\mathbf{d}}:=\left(\mathcal{F}_{0}^{\mathbf{d}} \subset \mathcal{F}_{1}^{\mathbf{d}} \subset \ldots \subset \mathcal{F}_{n}^{\mathbf{d}} \subset \ldots\right)$ defines a filtration on $U\left(\mathfrak{n}^{-}\right)$whose associated graded algebra is isomorphic to $S\left(\mathfrak{n}^{-}\right)$. 
(2) Let $V^{\mathbf{d}}(\lambda)$ be the graded module associated to the induced filtration. Then, $V^{\mathbf{d}}(\lambda)$ is a cyclic $S\left(\mathfrak{n}^{-}\right)$-module.

Proof. The universal enveloping algebra $U\left(\mathfrak{n}^{-}\right)$is a quotient of the tensor algebra $T\left(\mathfrak{n}^{-}\right)$by the ideal generated by $x \otimes y-y \otimes x-[x, y]$ for all $x, y \in \mathfrak{n}^{-}$. In $\mathfrak{n}^{-}$, for $\alpha, \beta, \gamma \in \Delta_{+}$with $\alpha+\beta=\gamma,\left[f_{\alpha}, f_{\beta}\right]$ is a multiple of $f_{\gamma}$; if $\mathbf{d} \in \mathcal{D}$, we have $d_{\alpha}+d_{\beta}>d_{\gamma}$, which proves the first part of the lemma. The second part is clear.

Let $v_{\lambda}^{\mathbf{d}}$ be a cyclic vector in $V^{\mathbf{d}}(\lambda)$. By (2) of the lemma, the $S\left(\mathfrak{n}^{-}\right)$-module map

$$
\varphi: S\left(\mathfrak{n}^{-}\right) \rightarrow V^{\mathbf{d}}(\lambda), \quad x \mapsto x \cdot v_{\lambda}^{\mathbf{d}}
$$

is surjective. We denote $I^{\mathbf{d}}(\lambda):=\operatorname{ker} \varphi$ and call it the defining ideal of $V^{\mathbf{d}}(\lambda)$.

2.3. The local and global monomial set. We are interested in some particular degrees such that the associated graded module admits "good" bases.

Definition 2.4. The local monomial set $\mathcal{S}_{\operatorname{lm}}$ is defined by

$$
\mathcal{S}_{\operatorname{lm}}:=\left\{\mathbf{d}=\left(d_{\beta}\right)_{\beta \in \Delta_{+}} \in S(\mathcal{D}) \mid \text { for any } i=1,2, \ldots, n, I^{\mathbf{d}}\left(\varpi_{i}\right) \text { is a monomial ideal }\right\} .
$$

REMARK 2.5. For any simple Lie algebra $\mathfrak{g}$, the local monomial set $\mathcal{S}_{\operatorname{lm}}$ is nonempty. For example, one possibility is to linearly order a monomial basis of any fixed regular representation. The induced order will be in the local monomial set.

DEFINITION 2.6. The global monomial set $\mathcal{S}_{\mathrm{gm}}$ is defined by

$$
\mathcal{S}_{\mathrm{gm}}:=\left\{\mathbf{d}=\left(d_{\beta}\right)_{\beta \in \Delta_{+}} \in S(\mathcal{D}) \mid \text { for any } \lambda \in \mathcal{P}_{+}, I^{\mathbf{d}}(\lambda) \text { is a monomial ideal }\right\} .
$$

It is clear that $\mathcal{S}_{\text {gm }} \subset \mathcal{S}_{\text {lm }}$.

The main goal of this paper is to study the following questions:

(1) Whether the global monomial set $\mathcal{S}_{\mathrm{gm}}$ is non-empty? That is to say, does there exist a filtration on $U\left(\mathfrak{n}^{-}\right)$arising from $\mathbf{d} \in \mathcal{D}$ such that for any finite-dimensional irreducible representation, the defining ideal of the associated graded module is monomial?

(2) If the answer to the above question is affirmative, for any $\lambda \in \mathcal{P}_{+}$, we obtain a unique monomial basis for $V^{\mathbf{d}}(\lambda)$ parameterized by $S(\lambda):=\left\{\mathbf{s} \in \mathbb{N}^{\Delta_{+}} \mid f^{\mathbf{s}} \cdot v_{\lambda}^{\mathbf{d}} \neq 0\right\}$. Whether there exists a lattice polytope $P(\lambda)$ such that $S(\lambda)$ is exactly the lattice points in $P(\lambda)$ ?

2.4. Criteria for the local monomial set. We first give a criterion to decide whether $\mathbf{d} \in \mathcal{D}$ is contained in $\mathcal{S}_{\text {lm }}$, which is useful in the rest of the paper.

We fix $\lambda \in \mathcal{P}_{+}$. For $\mu \in \mathcal{P}$ such that $r_{\mu}:=\operatorname{dim} V(\lambda)_{\mu} \neq 0$, we denote

$$
S_{\mu}:=\left\{\mathbf{s} \in \mathbb{N}^{\Delta_{+}} \mid f^{\mathbf{s}} \cdot v_{\lambda} \neq 0 \in V(\lambda) \text { and } \sum_{\alpha \in \Delta_{+}} s_{\alpha} \alpha=\lambda-\mu\right\}:
$$


this is a finite set. Suppose that $S_{\mu}=\left\{\mathbf{s}_{1}, \mathbf{s}_{2}, \ldots, \mathbf{s}_{m_{\mu}}\right\}$ with

$$
\operatorname{deg}_{\mathbf{d}}\left(f^{\mathbf{s}_{1}}\right) \leq \operatorname{deg}_{\mathbf{d}}\left(f^{\mathbf{s}_{2}}\right) \leq \ldots \leq \operatorname{deg}_{\mathbf{d}}\left(f^{\mathbf{s}_{m_{\mu}}}\right)
$$

Let $T_{\mu}=\left\{\mathbf{s}_{k} \mid f^{\mathbf{s}_{k}} \cdot v_{\lambda} \notin \operatorname{span}\left\{f^{\mathbf{s}_{1}} \cdot v_{\lambda}, \ldots, f^{\mathbf{s}_{k-1}} \cdot v_{\lambda}\right\}\right\}$. Then by construction, the set $\left\{f^{\mathbf{s}} . v_{\lambda} \mid \mathbf{s} \in T_{\mu}\right\}$ is a basis.

Lemma 2.7. Let $\mu \in \mathcal{P}$. Suppose

$$
\mathbf{s}_{k} \notin T_{\mu} \Rightarrow \operatorname{deg}\left(f^{\mathbf{s}_{k}}\right)>\operatorname{deg}\left(f^{\mathbf{s}_{l}}\right) \text { for all } \mathbf{s}_{l} \in T_{\mu} \text { with } l<k,
$$

then the defining ideal $I^{\mathbf{d}}(\lambda)$ is monomial.

Proof. It suffices to show that if $\mathbf{s}_{k} \notin T_{\mu}$, then $f^{\mathbf{s}_{k}} \in I^{\mathrm{d}}(\lambda)_{\mu}$. Indeed, by definition, $\mathbf{s}_{k} \notin T_{\mu}$ implies that $f^{\mathbf{s}_{k}} \cdot v_{\lambda}$ is a linear combination of $f^{\mathbf{s}_{i_{1}}} \cdot v_{\lambda}, \ldots, f^{\mathbf{s}_{i_{p}}} \cdot v_{\lambda}$ with $\mathbf{s}_{i_{q}} \in T_{\mu}$. By assumption, $\operatorname{deg}\left(f^{\mathbf{s}_{k}}\right)>\operatorname{deg}\left(f^{\mathbf{s}_{i_{q}}}\right)$, hence in the graded module, we have $f^{\mathbf{s}_{k}} \in I^{\mathbf{d}}(\lambda)_{\mu}$.

The following corollary is a special case of the lemma; it will be used repeatedly when dealing with the examples.

Corollary 2.8. The defining ideal $I^{\mathbf{d}}(\lambda)$ is monomial, if for any $\mu \in \mathcal{P}$ with $r_{\mu} \neq 0$ :

(1) if $r_{\mu}=1, \operatorname{deg}_{\mathbf{d}}\left(f^{\mathbf{s}_{1}}\right)<\operatorname{deg}_{\mathbf{d}}\left(f^{\mathbf{s}_{2}}\right)$;

(2) if $r_{\mu}>1, \#\left\{\operatorname{deg}_{\mathbf{d}}\left(f^{\mathbf{s}_{k}}\right) \mid 1 \leq k \leq m_{\mu}\right\}=m_{\mu}$.

2.5. How local and global monomial sets are related. We give a sufficient condition for an element in $\mathcal{S}_{\mathrm{lm}}$ being contained in $\mathcal{S}_{\mathrm{gm}}$. Let $\mathbf{d} \in \mathcal{S}_{\operatorname{lm}}$ and for $1 \leq i \leq n$, $S^{\mathbf{d}}\left(\varpi_{i}\right)=\left\{\mathbf{a} \in \mathbb{N}^{\Delta_{+}} \mid f^{\mathbf{a}} \cdot v_{\varpi_{i}}^{\mathbf{d}} \neq 0\right\}$. For an integer $m \geq 1$, let $S^{\mathbf{d}}\left(\varpi_{i}\right)^{+m}$ denote the $m$ fold Minkowski sum of $S^{\mathrm{d}}\left(\varpi_{i}\right)$. We will write $S\left(\varpi_{i}\right)$ to instead $S^{\mathrm{d}}\left(\varpi_{i}\right)$ when the context is clear.

THEOREM 2.9. For any $\lambda=m_{1} \varpi_{1}+m_{2} \varpi_{2}+\cdots+m_{n} \varpi_{n} \in \mathcal{P}_{+}$, if $\#\left(S\left(\varpi_{1}\right)^{+m_{1}}+\right.$ $\left.S\left(\varpi_{2}\right)^{+m_{2}}+\cdots+S\left(\varpi_{n}\right)^{+m_{n}}\right)=\operatorname{dim} V(\lambda)$, then $\mathbf{d} \in \mathcal{S}_{\mathrm{gm}}$.

The rest of this paragraph is devoted to the proof of this statement. This is based on the proof of [7, Theorem 3], which handles type $A$. For any $\tau=\sum_{i=1}^{n} r_{i} \varpi_{i} \in \mathcal{P}_{+}$, we define

$$
S(\tau):=S\left(\varpi_{1}\right)^{+r_{1}}+S\left(\varpi_{2}\right)^{+r_{2}}+\cdots+S\left(\varpi_{n}\right)^{+r_{n}} .
$$

We want to show simultaneously that for $\lambda, \mu \in \mathcal{P}_{+}$,

(1) $\left\{f^{\mathbf{s}} \cdot v_{\lambda+\mu}^{\mathbf{d}} \mid \mathbf{s} \in S(\lambda+\mu)\right\}$ is a basis of $V^{\mathbf{d}}(\lambda+\mu)$;

(2) the defining ideal $I^{\mathrm{d}}(\lambda+\mu)$ is monomial.

The statements will be proved by induction on the height of $\lambda+\mu$. The height 1 case is the assumption $\mathbf{d} \in \mathcal{S}_{\mathrm{Im}}$. The induction step will be divided into several parts.

Let $<$ be a total order on $\left\{f_{\beta} \mid \beta \in \Delta_{+}\right\}$refining the partial order defined by $\mathbf{d}=\left(d_{\beta}\right)_{\beta \in \Delta_{+}}$and consider the induced lexicographical order on the monomials in $U\left(\mathfrak{n}^{-}\right)$. The following proposition is proved essentially in [10, Proposition 2.11]; in [7, Proposition 4], it is proved in detail for a particular degree function for type $A_{n}$, but the proof used there is valid for a general $\mathbf{d}$. Both of the following two propositions are independent of the assumptions in Theorem 2.9. 
Proposition 2.10. For any $\lambda, \mu \in \mathcal{P}^{+}$the set $\left\{f^{\mathbf{s}} \cdot\left(v_{\lambda}^{\mathbf{d}} \otimes v_{\mu}^{\mathbf{d}}\right) \mid \mathbf{s} \in S(\lambda+\mu)\right\}$ is linear independent in $V^{\mathbf{d}}(\lambda) \otimes V^{\mathbf{d}}(\mu)$.

This set lies in the Cartan component of $V^{\mathbf{d}}(\lambda) \otimes V^{\mathbf{d}}(\mu)$ and since $|S(\lambda+\mu)|=$ $\operatorname{dim} V(\lambda+\mu)$ this set is a basis of the Cartan component of $V(\lambda) \otimes V(\mu)$ and of $V(\lambda+\mu)$, respectively.

Proposition 2.11. If $\mathbf{s} \notin S(\lambda+\mu)$, then $f^{\mathbf{s}} \cdot v_{\lambda+\mu}^{\mathbf{d}}=0$ in $V^{\mathbf{d}}(\lambda+\mu)$.

Proof. We fix s $\notin S(\lambda+\mu)$ and write

$$
f^{\mathbf{s}} \cdot v_{\lambda+\mu}=\sum_{\mathbf{t} \in S(\lambda+\mu)} c_{\mathbf{t}} f^{\mathbf{t}} \cdot v_{\lambda+\mu} \text { in } V(\lambda+\mu) .
$$

Since $V(\lambda+\mu) \subset V(\lambda) \otimes V(\mu)$, we have an expansion of the equation (1):

$$
f^{\mathbf{s}} \cdot\left(v_{\lambda} \otimes v_{\mu}\right)=\sum_{\mathbf{t} \in S(\lambda+\mu), \mathbf{a}+\mathbf{b}=\mathbf{t}} c_{\mathbf{t}} c_{\mathbf{a}, \mathbf{b}} f^{\mathbf{a}} \cdot v_{\lambda} \otimes f^{\mathbf{b}} \cdot v_{\mu} \text { in } V(\lambda) \otimes V(\mu)
$$

By replacing those $\mathbf{a} \notin S(\lambda)($ resp. $\mathbf{b} \notin S(\mu))$ by a sum supported on $S(\lambda)($ resp. $S(\mu))$, we obtain a unique expression. By induction, the corresponding monomials have strictly lower degrees then $\operatorname{deg}\left(f^{\mathbf{a}}\right)\left(\operatorname{resp} . \operatorname{deg}\left(f^{\mathbf{b}}\right)\right)$. This implies that we have for all $\mathbf{t}$ appearing in this unique expression $\operatorname{deg}\left(f^{\mathbf{t}}\right)<\operatorname{deg}\left(f^{\mathbf{s}}\right)$.

Proposition 2.12. Suppose the assumptions of Theorem 2.9 are satisfied, then the set $\mathcal{B}=\left\{f^{\mathbf{s}} \cdot v_{\lambda+\mu}^{\mathbf{d}} \mid \mathbf{s} \in S(\lambda+\mu)\right\}$ is a basis of $V^{\mathbf{d}}(\lambda+\mu)$.

Proof. By considering each filtration component, this is a direct consequence of Proposition 2.11.

We are left with proving (2), the monomiality of the annihilating ideal. This follows immediately from the following proposition.

Proposition 2.13. Suppose the assumptions of Theorem 2.9 are satisfied, then the defining ideal of the Cartan component of $V^{\mathbf{d}}(\lambda) \otimes V^{\mathbf{d}}(\mu)$ is monomial and there exists an $S\left(\mathfrak{n}^{-}\right)$-module isomorphism from the Cartan component of $V^{\mathbf{d}}(\lambda) \otimes V^{\mathbf{d}}(\mu)$ to $V^{\mathbf{d}}(\lambda+\mu)$.

Proof. We have for $\mathbf{s} \notin S(\lambda+\mu)=S(\lambda)+S(\mu)$ :

$$
f^{\mathbf{s}} \cdot\left(v_{\lambda} \otimes v_{\mu}\right)=\sum_{\mathbf{t}_{1}+\mathbf{t}_{2}=\mathbf{s}} f^{\mathbf{t}_{1}} \cdot v_{\lambda} \otimes f^{\mathbf{t}_{2}} \cdot v_{\mu} \text { in } V(\lambda) \otimes V(\mu)
$$

and $\mathbf{t}_{1} \notin S(\lambda)$ or $\mathbf{t}_{2} \notin S(\mu)$. Hence, by Proposition 2.11, we can conclude that either $f^{\mathbf{t}_{1}} \cdot v_{\lambda}^{\mathbf{d}}=0$ in $V^{\mathbf{d}}(\lambda)$ or $f^{\mathbf{t}_{2}} \cdot v_{\mu}^{\mathbf{d}}=0$ in $V^{\mathbf{d}}(\mu)$. We obtain

$$
f^{\mathbf{s}} \cdot\left(v_{\lambda}^{\mathbf{d}} \otimes v_{\mu}^{\mathbf{d}}\right)=0 \text { in } V^{\mathbf{d}}(\lambda) \otimes V^{\mathbf{d}}(\mu) .
$$

Therefore, we have monomiality.

By Proposition 2.11, there is a surjective map of $S\left(\mathfrak{n}^{-}\right)$-modules from the Cartan component of $V^{\mathbf{d}}(\lambda) \otimes V^{\mathbf{d}}(\mu)$ to $V^{\mathbf{d}}(\lambda+\mu)$, which is an isomorphism for dimension reasons.

This proves the monomiality statement (2) and hence Theorem 2.9 , i.e. $\mathbf{d} \in \mathcal{S}_{\mathrm{gm}}$. 


\section{Quantum groups and quantum degree cones.}

3.1. Quantum groups. Let $C=\left(c_{i j}\right)_{n \times n} \in \operatorname{Mat}_{n}(\mathbb{Z})$ be the Cartan matrix of $\mathfrak{g}$ and $D=\operatorname{diag}\left(d_{1}, \ldots, d_{n}\right) \in \operatorname{Mat}_{n}(\mathbb{Z})$ be a diagonal matrix symmetrizing $C$. Thus, $A=D C=\left(a_{i j}\right)_{n \times n} \in \operatorname{Mat}_{n}(\mathbb{Z})$ is symmetric. Let $U_{q}(\mathfrak{g})$ be the corresponding quantum group over $\mathbb{C}(q)$ : As an algebra, it is generated by $E_{i}, F_{i}$ and $K_{i}^{ \pm 1}$ for $i=1, \ldots, n$, subject to the following relations: For $i, j=1, \ldots, n$,

$$
\begin{gathered}
K_{i} K_{i}^{-1}=K_{i}^{-1} K_{i}=1, \quad K_{i} E_{j} K_{i}^{-1}=q_{i}^{c_{i j}} E_{j}, \quad K_{i} F_{j} K_{i}^{-1}=q_{i}^{-c_{i j}} F_{j}, \\
E_{i} F_{j}-F_{j} E_{i}=\delta_{i j} \frac{K_{i}-K_{i}^{-1}}{q_{i}-q_{i}^{-1}},
\end{gathered}
$$

and for $i \neq j$,

$$
\sum_{r=0}^{1-c_{i j}}(-1)^{r} E_{i}^{\left(1-c_{i j}-r\right)} E_{j} E_{i}^{(r)}=0, \quad \sum_{r=0}^{1-c_{i j}}(-1)^{r} F_{i}^{\left(1-c_{i j}-r\right)} F_{j} F_{i}^{(r)}=0,
$$

where

$$
q_{i}=q^{d_{i}},[n]_{q} !=\prod_{i=1}^{n} \frac{q^{n}-q^{-n}}{q-q^{-1}}, \quad E_{i}^{(n)}=\frac{E_{i}^{n}}{[n]_{q_{i}} !} \text { and } F_{i}^{(n)}=\frac{F_{i}^{n}}{[n]_{q_{i}} !} .
$$

Let $U_{q}\left(\mathfrak{n}^{-}\right)$be the sub-algebra of $U_{q}(\mathfrak{g})$ generated by $F_{i}$ for $i=1, \ldots, n$. For $\lambda \in \mathcal{P}_{+}$, we denote by $V_{q}(\lambda)$ the finite-dimensional irreducible representation of $U_{q}(\mathfrak{g})$ of highest weight $\lambda$ and type 1 with highest weight vector $\mathbf{v}_{\lambda}$.

When $q$ is specialized to 1 , the quantum group $U_{q}(\mathfrak{g})$ admits $U(\mathfrak{g})$ as its classical limit. In this limit, the representation $V_{q}(\lambda)$ is specialized to $V(\lambda)$.

3.2. PBW root vectors and commutation relations. Let $T_{i}=T_{i, 1}^{\prime \prime}, i=1, \ldots, n$ be Lusztig's automorphisms:

$$
T_{i}\left(E_{i}\right)=-F_{i} K_{i}, \quad T_{i}\left(F_{i}\right)=-K_{i}^{-1} E_{i}, \quad T_{i}\left(K_{j}\right)=K_{j} K_{i}^{-c_{i j}},
$$

for $i=1, \ldots, n$, and $j \neq i$,

$$
T_{i}\left(E_{j}\right)=\sum_{r+s=-c_{i j}}(-1)^{r} q_{i}^{-r} E_{i}^{(s)} E_{j} E_{i}^{(r)}, \quad T_{i}\left(F_{j}\right)=\sum_{r+s=-c_{i j}}(-1)^{r} q_{i}^{r} F_{i}^{(r)} F_{j} F_{i}^{(s)} .
$$

For details, see Chapter 37 in [19]. We fix a reduced decomposition $\underline{w}_{0}=s_{i_{1}} \ldots s_{i_{N}} \in$ $R\left(w_{0}\right)$ and let positive roots $\beta_{1}, \beta_{2}, \ldots, \beta_{N}$ be defined as in Section 2.1. The quantum PBW root vector $F_{\beta_{t}}$ associated to a positive root $\beta_{t}$ is defined by

$$
F_{\beta_{t}}=T_{i_{1}} T_{i_{2}} \ldots T_{i_{t-1}}\left(F_{i_{t}}\right) \in U_{q}\left(\mathfrak{n}^{-}\right) .
$$

The PBW theorem of quantum groups affirms that the set

$$
\left\{F^{\mathbf{s}}:=F_{\beta_{1}}^{s_{1}} F_{\beta_{2}}^{s_{2}} \ldots F_{\beta_{N}}^{s_{N}} \mid \mathbf{s}=\left(s_{1}, \ldots, s_{N}\right) \in \mathbb{N}^{N}\right\}
$$

forms a $\mathbb{C}(q)$-basis of $U_{q}\left(\mathfrak{n}^{-}\right)$([19], Corollary 40.2.2). 
The commutation relations between these quantum PBW root vectors are given by the following $\mathrm{L}-\mathrm{S}$ formula: For any $i<j$,

$$
F_{\beta_{j}} F_{\beta_{i}}-q^{-\left(\beta_{i}, \beta_{j}\right)} F_{\beta_{i}} F_{\beta_{j}}=\sum_{n_{i+1}, \ldots, n_{j-1} \geq 0} c\left(n_{i+1}, \ldots, n_{j-1}\right) F_{\beta_{i+1}}^{n_{i+1}} \ldots F_{\beta_{j-1}}^{n_{j-1}},
$$

where $c\left(n_{i+1}, \ldots, n_{j-1}\right) \in \mathbb{C}\left[q^{ \pm 1}\right]$. We denote

$$
M_{i, j}=\left\{F_{\beta_{i+1}}^{n_{i+1}} F_{\beta_{i+2}}^{n_{i+2}} \ldots F_{\beta_{j-1}}^{n_{j-1}} \mid n_{i+1} \beta_{i+1}+n_{i+2} \beta_{2}+\cdots+n_{j-1} \beta_{j-1}=\beta_{i}+\beta_{j}\right\},
$$

then for weight reasons, the sum in the right-hand side of (3) is supported inside $M_{i, j}$. Denote by $M_{i, j}^{q} \subset M_{i, j}$, the set of monomials which actually appear with a non-zero coefficient in the right-hand side of (3). It should be pointed out that the right-hand side of (3) largely depends on the chosen reduced decomposition. In general, it is hard to know which monomials appear in $M_{i, j}^{q}$.

Let us have a closer look on how these formulas depend on the reduced decomposition. Let $\underline{w}_{0}, \underline{w}_{0}^{\prime} \in R\left(w_{0}\right)$ be two reduced decompositions such that they are of form:

$$
\underline{w}_{0}=\underline{w}_{L} s_{p} s_{q} \underline{w}_{R}, \quad \underline{w}_{0}^{\prime}=\underline{w}_{L} s_{q} s_{p} \underline{w}_{R}
$$

with $1 \leq p \neq q \leq n$ and $s_{p} s_{q}=s_{q} s_{p}$. We define $l=\ell\left(\underline{w}_{L}\right)$.

Let the convex total order on $\Delta_{+}$induced by $\underline{w}_{0}\left(\right.$ resp. $\left.\underline{w}_{0}^{\prime}\right)$ be

$$
\beta_{1}<\beta_{2}<\ldots<\beta_{N}\left(\text { resp. } \beta_{1}^{\prime}<\beta_{2}^{\prime}<\ldots<\beta_{N}^{\prime}\right) .
$$

For $s \leq l$, the $\mathrm{L}-\mathrm{S}$ formula (3) reads

$$
F_{\beta_{s}} F_{\beta_{l+2}}-q^{\left(\beta_{s}, \beta_{l+2}\right)} F_{\beta_{l+2}} F_{\beta_{s}}=\sum_{n_{s+1}, \ldots, n_{l+1} \geq 0} c\left(n_{s+1}, \ldots, n_{l+1}\right) F_{\beta_{s+1}}^{n_{s+1}} \ldots F_{\beta_{l+1}}^{n_{l+1}} .
$$

For $t \geq l+3$, the $\mathrm{L}-\mathrm{S}$ formula (3) reads

$$
F_{\beta_{t}} F_{\beta_{l+1}}-q^{-\left(\beta_{t}, \beta_{l+1}\right)} F_{\beta_{l+1}} F_{\beta_{t}}=\sum_{n_{l+2}, \ldots, n_{t-1} \geq 0} c\left(n_{l+2}, \ldots, n_{t-1}\right) F_{\beta_{l+2}}^{n_{l+2}} \ldots F_{\beta_{t-1}}^{n_{t-1}} .
$$

LEMMA 3.1. In the formula (4), $n_{l+1}=0$; in the formula (5), $n_{l+2}=0$.

Proof. We prove for example the first statement, the second one can be shown similarly.

First, notice that for any $i \neq l+1, l+2, \beta_{i}=\beta_{i}^{\prime}, \beta_{l+1}=\beta_{l+2}^{\prime}, \beta_{l+2}=\beta_{l+1}^{\prime}$. The same argument can be applied to quantum PBW root vectors: Let $F_{\beta_{1}}, F_{\beta_{2}}, \ldots, F_{\beta_{N}}$ (resp. $F_{\beta_{1}}^{\prime}, F_{\beta_{2}}^{\prime}, \ldots, F_{\beta_{N}}^{\prime}$ ) be the quantum PBW root vectors obtained from $\underline{w}_{0}$ (resp. $\left.\underline{w}_{0}^{\prime}\right)$. Then, for any $i \neq l+1, l+2, F_{\beta_{i}}=F_{\beta_{i}}^{\prime}, F_{\beta_{l+1}}=F_{\beta_{l+2}}^{\prime}, F_{\beta_{l+2}}=F_{\beta_{l+1}}^{\prime}$. For $s \leq l$, we apply the $\mathrm{L}-\mathrm{S}$ formula to $F_{\beta_{s}}^{\prime}$ and $F_{\beta_{l+1}}^{\prime}$, it gives

$$
F_{\beta_{s}}^{\prime} F_{\beta_{l+1}}^{\prime}-q^{\left(\beta_{s}^{\prime}, \beta_{l+1}^{\prime}\right)} F_{\beta_{l+1}}^{\prime} F_{\beta_{s}}^{\prime}=\sum_{m_{s+1}, \ldots, m_{l} \geq 0} d\left(m_{s+1}, \ldots, m_{l}\right) F_{\beta_{s+1}}^{\prime m_{s+1}} \ldots F_{\beta_{l}}^{\prime m_{l}} .
$$

Comparing it to (4) gives $n_{l+1}=0$. 
3.3. Quantum degree cones. We fix in this paragraph a reduced decomposition $\underline{w}_{0} \in R\left(w_{0}\right)$ and positive roots $\beta_{1}, \ldots, \beta_{N}$ obtained from $\underline{w}_{0}$ as explained in Section 2.1 .

DEFINITION 3.2. The quantum degree cone associated to $\underline{w}_{0}$ is defined by

$$
\mathcal{D}_{\underline{w}_{0}}^{q}:=\left\{\left(d_{\beta}\right)_{\beta \in \Delta_{+}} \in \mathbb{R}_{\geq 0}^{\Delta_{+}} \mid \forall i<j, d_{\beta_{i}}+d_{\beta_{j}}>\sum_{k=i+1}^{j-1} n_{k} d_{\beta_{k}} \text { if } c\left(n_{i+1}, \ldots, n_{j-1}\right) \neq 0 \text { in (3) }\right\} .
$$

We denote the set

$$
\mathcal{D}^{q}:=\bigcup_{\underline{w}_{0} \in R\left(w_{0}\right)} \mathcal{D}_{\underline{w}_{0}}^{q} \subset \mathbb{R}_{\geq 0}^{\Delta_{+}}
$$

Let $\mathcal{D} \subset \mathbb{R}_{\geq 0}^{\Delta_{+}}$be the classical degree cone. Specializing the quantum parameter $q$ to 1 proves the following lemma:

LEMMA 3.3. We have $\mathcal{D}^{q} \subset \mathcal{D}$.

REMARK 3.4. Except for small rank cases $\mathfrak{g}=\mathfrak{s l}_{2}, \mathfrak{s l}_{3}$ (see Example 4.1), the inclusion in Lemma 3.3 is strict. For example, the constant function 1 taking value 1 on each positive root is in the classical degree cone $\mathcal{D}$, but for $\mathfrak{g} \neq \mathfrak{s l}_{2}, \mathfrak{s l}_{3}$, there is no reduced decomposition $\underline{w}_{0}$ such that $\mathbf{1} \notin \mathcal{D}_{\underline{w}_{0}}^{q}$. See for example [7, Section 2.4] and Example 4.2 for type $\mathrm{C}_{2}$, Section 4.3 for type $\mathrm{G}_{2}$.

Let $\mathbf{d}=\left(d_{\beta}\right)_{\beta \in \Delta_{+}} \in S\left(\mathcal{D}_{\underline{w}_{0}}^{q}\right)=: \mathcal{D}_{\underline{w}_{0}}^{q} \cap \mathbb{N}^{\Delta_{+}}$. For a monomial $F^{\mathbf{t}}$ where $\mathbf{t}=$ $\left(t_{1}, \ldots, t_{N}\right)$, we define its d-degree $\operatorname{deg}_{\mathbf{d}}$ by

$$
\operatorname{deg}_{\mathbf{d}}\left(F^{\mathbf{t}}\right):=t_{1} d_{\beta_{1}}+t_{2} d_{\beta_{2}}+\cdots+t_{N} d_{\beta_{N}} .
$$

THEOREM 3.5. The set $\mathcal{D}_{\underline{w}_{0}}^{q}$ is a non-empty open polyhedral cone.

Proof. By definition, $\mathcal{D}_{\underline{w}_{0}}^{q}$ is an open polyhedral cone. We describe an inductive procedure to construct an element $\mathbf{d}=\left(d_{\beta_{1}}, \ldots, d_{\beta_{N}}\right) \in \mathcal{D}_{\underline{w}_{0}}^{q}$.

We set $d_{\beta_{1}}=1$. Suppose that $d_{\beta_{1}}, \ldots, d_{\beta_{k}}$ are chosen such that they satisfy the inequalities in the definition of $\mathcal{D}_{\underline{w}_{0}}^{q}$.

Let $M_{k+1}^{q}:=\bigcup_{s=1}^{k} M_{s, k+1}^{q}$. Since $M_{k+1}^{q}$ is a finite set, we set

$$
d_{\beta_{k+1}}=1+\max _{F^{\mathbf{t}} \in M_{k+1}^{q}}\left(\operatorname{deg}_{\mathbf{d}}\left(F^{\mathbf{t}}\right)\right) .
$$

Since $F^{\mathbf{t}} \in M_{k+1}^{q}$ is a monomial on $\left\{F_{\beta_{1}}, \ldots, F_{\beta_{k}}\right\}$, the degree is well-defined. By definition, for any $1 \leq s \leq k$ and any $F^{\mathbf{t}} \in M_{s, k+1}^{q}, \quad d_{\beta_{s}}+d_{\beta_{k+1}}>\operatorname{deg}_{\mathbf{d}}\left(F^{\mathbf{t}}\right)$. This terminates the proof. by

For $\mathbf{d} \in S\left(\mathcal{D}_{\underline{w}_{0}}^{q}\right)$, we define a filtration $\mathcal{F}_{\bullet}^{\mathbf{d}}=\left(\mathcal{F}_{0}^{\mathbf{d}} \subset \mathcal{F}_{1}^{\mathbf{d}} \subset \ldots \subset \mathcal{F}_{n}^{\mathbf{d}} \subset \ldots\right)$ on $U_{q}\left(\mathfrak{n}^{-}\right)$

$$
\mathcal{F}_{k}^{\mathbf{d}} U_{q}\left(\mathfrak{n}^{-}\right):=\operatorname{span}\left\{F^{\mathbf{t}} \mid \operatorname{deg}_{\mathbf{d}}\left(F^{\mathbf{t}}\right) \leq k\right\}
$$


Let $S_{q}\left(\mathfrak{n}^{-}\right)$be the algebra generated by $x_{1}, x_{2}, \ldots, x_{N}$, subjects to the following relations: For $1 \leq i<j \leq N$,

$$
x_{i} x_{j}=q^{\left(\beta_{i}, \beta_{j}\right)} x_{j} x_{i} .
$$

The following proposition is clear from the $\mathrm{L}-\mathrm{S}$ formula (3).

PRoposition 3.6.

(1) The filtration $\mathcal{F}_{\bullet}$ endows $U_{q}\left(\mathfrak{n}^{-}\right)$with a filtered algebra structure.

(2) The associated graded algebra $\operatorname{gr}_{\mathcal{F}} U_{q}\left(\mathfrak{n}^{-}\right)$is isomorphic to $S_{q}\left(\mathfrak{n}^{-}\right)$.

For $\lambda \in \mathcal{P}_{+}$, the above filtration on $U_{q}\left(\mathfrak{n}^{-}\right)$induces a filtration on $V_{q}(\lambda)$ by letting

$$
\mathcal{F}_{k}^{\mathrm{d}} V_{q}(\lambda):=\mathcal{F}_{k}^{\mathrm{d}} U_{q}\left(\mathfrak{n}^{-}\right) \cdot \mathbf{v}_{\lambda} .
$$

We let $V_{q}^{\mathrm{d}}(\lambda)$ denote the associated graded vector space: It is a cyclic $S_{q}\left(\mathfrak{n}^{-}\right)$-module. Let $\mathbf{v}_{\lambda}^{\mathbf{d}}$ be the cyclic vector corresponding to $\mathbf{v}_{\lambda}$.

\section{Examples of rank 2 and properties of quantum degree cones.}

4.1. Examples of rank 2. Before studying properties of these cones, we examine some small rank examples.

EXAMPLE 4.1. Let $\mathfrak{g}=\mathfrak{s} \mathfrak{l}_{3}$ be the Lie algebra of type $\mathrm{A}_{2}$. For $\mathbf{d} \in \mathcal{D}$, let $d_{i, j}=\mathbf{d}\left(\alpha_{i, j}\right)$.

We fix a sequence of positive roots $\left(\alpha_{1,1}, \alpha_{1,2}, \alpha_{2,2}\right)$. The classical degree cone $\mathcal{D}$ is given by

$$
\mathcal{D}=\left\{\left(d_{1,1}, d_{1,2}, d_{2,2}\right) \in \mathbb{R}_{\geq 0}^{\Delta_{+}} \mid d_{1,1}+d_{2,2}>d_{1,2}\right\} .
$$

We consider the quantum degree cones: $R\left(w_{0}\right)=\left\{s_{1} s_{2} s_{1}, s_{2} s_{1} s_{2}\right\}$. For the reduced decomposition $\underline{w}_{0}=s_{1} s_{2} s_{1}$, let $F_{1,1}, F_{1,2}, F_{2,2}$ be the corresponding quantum PBW root vectors. The formula (3) reads

$$
F_{1,1} F_{2,2}=q^{-1} F_{2,2} F_{1,1}-q^{-1} F_{1,2},
$$

implying $\mathcal{D}_{\underline{w}_{0}}^{q}=\mathcal{D}$. For $\underline{w}_{0}^{\prime}=s_{2} s_{1} s_{2}$, the same computation shows that $\mathcal{D}_{\underline{w}_{0}^{\prime}}^{q}=\mathcal{D}$.

EXAMPLE 4.2. Let $\mathfrak{g}=\mathfrak{s p}_{4}$ be the Lie algebra of type $\mathrm{C}_{2}$.

For $\mathbf{d} \in \mathcal{D}$, let $d_{i, j}:=\mathbf{d}\left(\alpha_{i, j}\right)$ and $d_{i, \bar{j}}:=\mathbf{d}\left(\alpha_{i, j}\right)$. The classical degree cone $\mathcal{D}$ is given by the following inequalities in $\mathbb{R}_{\geq 0}^{\Delta_{+}}$:

$$
d_{1,1}+d_{2,2}>d_{1,2}, \quad d_{1,1}+d_{1,2}>d_{1, \overline{1}} .
$$

Fix a reduced decomposition $\underline{w}_{0}=s_{1} s_{2} s_{1} s_{2}$ of the longest element $w_{0}$ in the Weyl group of $\mathfrak{g}$. Let

$$
F_{1,1}, \quad F_{1, \overline{1}}, \quad F_{1,2}, \quad F_{2,2}
$$

be the corresponding quantum PBW root vectors, their commutation relations are

$$
\begin{aligned}
& F_{1,1} F_{1, \overline{1}}=q^{2} F_{1, \overline{1}} F_{1,1}, \quad F_{1,1} F_{1,2}=F_{1,2} F_{1,1}-\left(q+q^{-1}\right) F_{1, \overline{1}}, \\
& F_{1,1} F_{2,2}=q^{-2} F_{2,2} F_{1,1}-q^{-2} F_{1,2},
\end{aligned}
$$




$$
F_{1, \overline{1}} F_{1,2}=q^{2} F_{1,2} F_{1, \overline{1}}, \quad F_{1, \overline{1}} F_{2,2}=F_{2,2} F_{1, \overline{1}}+\left(1-q^{-2}\right) F_{1,2}^{(2)}, \quad F_{1,2} F_{2,2}=q^{2} F_{2,2} F_{1,2} .
$$

The quantum degree cone $\mathcal{D}_{\underline{w}_{0}}^{q} \subset \mathcal{D}$ is given by

$$
d_{1,1}+d_{2,2}>d_{1,2}, \quad d_{1,1}+d_{1,2}>d_{1, \overline{1}}, \quad d_{2,2}+d_{1, \overline{1}}>2 d_{1,2} .
$$

The same construction with the reduced decomposition $\underline{w}_{0}^{\prime}=s_{2} s_{1} s_{2} s_{1}$ shows that $\mathcal{D}_{\underline{w}_{0}}^{q}=\mathcal{D}_{\underline{w}_{0}^{\prime}}^{q}$.

EXAMPLE 4.3. Let $\mathfrak{g}$ be the Lie algebra of type $\mathrm{G}_{2}$ with positive roots

$$
\Delta_{+}=\left\{\alpha_{1}, \alpha_{2}, \alpha_{1}+\alpha_{2}, 2 \alpha_{1}+\alpha_{2}, 3 \alpha_{1}+\alpha_{2}, 3 \alpha_{1}+2 \alpha_{2}\right\}
$$

For $\mathbf{d} \in \mathbb{R}_{>0}^{\Delta_{+}}$, we write $d_{1}=\mathbf{d}\left(\alpha_{1}\right), d_{2}=\mathbf{d}\left(\alpha_{2}\right), d_{12}=\mathbf{d}\left(\alpha_{1}+\alpha_{2}\right), d_{112}=\mathbf{d}\left(2 \alpha_{1}+\alpha_{2}\right)$, $d_{1112}=\mathbf{d}\left(3 \alpha_{1}+\alpha_{2}\right)$ and $d_{11122}=\mathbf{d}\left(3 \alpha_{1}+2 \alpha_{2}\right)$. The classical degree cone $\mathcal{D} \subset \mathbb{R}_{\geq 0}^{\Delta_{+}}$is determined by the following inequalities:

$$
\begin{gathered}
d_{1}+d_{2}>d_{12}, \quad d_{1}+d_{12}>d_{112}, \quad d_{1}+d_{112}>d_{1112}, \\
d_{2}+d_{1112}>d_{11122}, \quad d_{112}+d_{12}>d_{11122} .
\end{gathered}
$$

For example, $\left(d_{1}, d_{1112}, d_{112}, d_{11122}, d_{12}, d_{2}\right)=(2,1,3,1,3,2) \in \mathcal{D}$.

We fix a reduced decomposition $\underline{w}_{0}=s_{1} s_{2} s_{1} s_{2} s_{1} s_{2} \in R\left(w_{0}\right)$. Let

$$
F_{1}, F_{1112}, F_{112}, F_{11122}, F_{12}, F_{2}
$$

be the corresponding quantum PBW root vectors. The quantum degree cone $\mathcal{D}_{\underline{w}_{0}}^{q}$ in $\mathcal{D}$ is given by the following inequalities:

$$
\begin{array}{ccc}
d_{1}+d_{11122}>2 d_{112}, & d_{1112}+d_{11122}>3 d_{112}, & d_{1112}+d_{12}>2 d_{112}, \\
d_{1112}+d_{2}>d_{112}+d_{12}, & d_{112}+d_{2}>2 d_{12}, & d_{11122}+d_{2}>3 d_{12}
\end{array}
$$

These inequalities are the same for the other reduced decomposition $\underline{w}_{0}^{\prime}=$ $s_{2} s_{1} s_{2} s_{1} s_{2} s_{1} \in R\left(w_{0}\right)$. It is clear that $\mathbf{d} \notin \mathcal{D}_{\underline{w}_{0}}^{q}$ for any $\underline{w}_{0} \in R\left(w_{0}\right)$, we see once again that the quantum degree cone is different from the classical degree cone.

In the rank 2 case, the quantum degree cone does not depend on the reduced decomposition.

Proposition 4.4. Let $\mathfrak{g}$ be a simple Lie algebra of rank no more than 2. For any $\underline{w}_{0} \in R\left(w_{0}\right)$, we have $\mathcal{D}^{q}=\mathcal{D}_{\underline{w}_{0}}^{q}$.

Proof. The proof follows from the preceeding examples.

4.2. Properties of quantum degree cones. The first property of the quantum degree cones we will prove is the following:

THEOREM 4.5. Let $\mathfrak{g}$ be a simple Lie algebra of rank $n \geq 3$, then

$$
\bigcap_{\underline{w}_{0} \in R\left(w_{0}\right)} \mathcal{D}_{\underline{w}_{0}}^{q}=\emptyset .
$$


Proof. We show that there exist two reduced decompositions $\underline{w}_{0}^{1}, \underline{w}_{0}^{2} \in R\left(w_{0}\right)$ such that $\mathcal{D}_{\underline{w}_{0}^{1}}^{q} \cap \mathcal{D}_{\underline{w}_{0}^{2}}^{q}=\emptyset$. When $\mathfrak{g}$ is a simple Lie algebra $\mathfrak{g}$ of rank 3 , this is proved in Section $6.1-6.3$ by explicit constructions.

Let $\mathfrak{g}$ be a simple Lie algebra of rank $>3$. There exists a Lie sub-algebra $\mathfrak{g}^{\prime} \subset \mathfrak{g}$ of rank 3 such that $\mathfrak{g}^{\prime}$ is a simple Lie algebra, we denote it by $X_{3}$. The set of positive roots of $\mathrm{x}_{3}$ is denoted by $\Delta_{+}^{\prime}$. We take $\underline{w}_{L}$ and $\underline{w}_{L}^{\prime}$ as in the example of $\mathrm{X}_{3}$ in Section 6.1-6.3, such that $\mathcal{D}_{\underline{w}_{L}}^{q} \cap \mathcal{D}_{\underline{w}_{L}^{\prime}}^{q}=\emptyset$. Let $\underline{w}_{0}^{1}=\underline{w}_{L} \underline{w}_{R}$ and $\underline{w}_{0}^{2}=\underline{w}_{L}^{\prime} \underline{w}_{R}^{\prime} \in R\left(w_{0}\right)$. We claim that $\mathcal{D}_{\underline{w}_{0}^{1}}^{q} \cap \mathcal{D}_{\underline{w}_{0}^{2}}^{q}=\emptyset$. Let $p: \mathcal{D}_{\underline{w}_{0}^{1}}^{q} \rightarrow \mathbb{R}_{\geq 0}^{\Delta_{+}^{\prime}}$ be the restriction of functions. Then by definition,

$$
p\left(\mathcal{D}_{\underline{w}_{0}^{1}}^{q}\right)=\mathcal{D}_{\underline{w}_{L}}^{q}, \quad p\left(\mathcal{D}_{\underline{w}_{0}^{2}}^{q}\right)=\mathcal{D}_{\underline{w}_{L}^{\prime}}^{q} .
$$

This terminates the proof.

This theorem implies that there is no degree function working for all reduced decompositions. In general, to study the relations between the cones associated to $\underline{w}_{0}$ and $\underline{w}_{0}^{\prime} \in R\left(w_{0}\right)$ is a difficult task. But in some cases, the cone remain the same.

Two reflections $s_{p}$ and $s_{q}$ in $W$ with $p \neq q$ are said to be orthogonal if $s_{p} s_{q}=s_{q} s_{p}$. Two reduced decompositions $\underline{w}_{0}, \underline{w}_{0}^{\prime} \in R\left(w_{0}\right)$ are said to be related by orthogonal reflections if one can be obtained from the other by using only orthogonal reflections.

Proposition 4.6. Let $\underline{w}_{0}, \underline{w}_{0}^{\prime} \in R\left(w_{0}\right)$ such that they are related by orthogonal reflections. Then, $\mathcal{D}_{\underline{w}_{0}}^{q}=\mathcal{D}_{\underline{w}_{0}^{\prime}}^{q}$.

Proof. By definition, it suffices to consider the case where

$$
\underline{w}_{0}=\underline{w}_{L} s_{p} s_{q} \underline{w}_{R}, \quad \underline{w}_{0}^{\prime}=\underline{w}_{L} s_{q} s_{p} \underline{w}_{R}
$$

with $1 \leq p, q \leq n$ such that $s_{p} s_{q}=s_{q} s_{p}$. In this case, Lemma 3.1 can be applied to finish the proof.

\section{Local and global monomial sets.}

5.1. Local monomial set for type $A_{n}$. Let $\mathfrak{g}=\mathfrak{s l}_{n+1}$ be the Lie algebra of type $A_{n}$.

The following lemma gives an easy criterion to determine whether a degree is in the local monomial set.

Proposition 5.1. Let $\mathbf{d} \in S(\mathcal{D})$. The following statements are equivalent:

(1) For any four different positive roots $\alpha, \beta, \gamma, \delta$ satisfying $\alpha+\beta=\gamma+\delta, d_{\alpha}+d_{\beta} \neq$ $d_{\gamma}+d_{\delta}$

(2) $\mathbf{d} \in \mathcal{S}_{\mathrm{lm}}$.

Proof. (1) $\Rightarrow(2)$ : Since in the $A_{n}$ case, all fundamental representations are minuscule. The proof of [7, Proposition 2] can be applied to show the validity of the hypothesis of Corollary 2.8 .

(2) $\Rightarrow(1)$ : Since $\mathfrak{g}=\mathfrak{s l}_{n+1}$, we can suppose that $\alpha=\alpha_{i, j}, \beta=\alpha_{k, l}, \gamma=\alpha_{i, l}$ and $\delta=\alpha_{j, k}$ with $i \leq k \leq j \leq l$. We consider the fundamental representation $V\left(\varpi_{l}\right)$ : $\operatorname{In} I\left(\varpi_{l}\right)$, there is a relation $f_{i, j} f_{k, l} v_{\varpi_{l}} \pm f_{i, l} f_{k, j} v_{\varpi_{l}}=0$. Since $I^{\mathbf{d}}\left(\varpi_{l}\right)$ is monomial, either $f_{i, j} f_{k, l}$ or $f_{i, l} f_{k, j}$ is in $I^{\mathrm{d}}\left(\varpi_{l}\right)$, which forbids the case $d_{\alpha_{i, j}}+d_{\alpha_{k, l}}=d_{\alpha_{i, l}}+d_{\alpha_{k, j}}$. 
EXAMPLE 5.2. In general, the inclusions $\mathcal{D}_{\underline{w}_{0}}^{q} \subset \mathcal{S}_{\operatorname{lm}}$ and $\mathcal{S}_{\operatorname{lm}} \subset \mathcal{D}^{q}$ do not hold. Let $\mathfrak{g}$ be of type $A_{3}$. The reduced decomposition $\underline{w}_{0}=s_{1} s_{2} s_{3} s_{2} s_{1} s_{2} \in R\left(w_{0}\right)$ induces the convex order on $\Delta_{+}$:

$$
\alpha_{1,1}<\alpha_{1,2}<\alpha_{1,3}<\alpha_{3,3}<\alpha_{2,3}<\alpha_{2,2} \text {. }
$$

We fix this sequence and identify $\mathbb{N}^{\Delta_{+}}$with $\mathbb{N}^{6}$. Let $\mathbf{d}=(1,1,1,1,1,1)$, $\mathbf{d}^{\prime}=$ $(2,2,1,1,1,1)$ and $\mathbf{d}^{\prime \prime}=(1,1,1,1,1,2)$. By Proposition 5.1, $\mathbf{d} \notin \mathcal{S}_{\mathrm{lm}}, \mathbf{d}^{\prime}, \mathbf{d}^{\prime \prime} \in \mathcal{S}_{\mathrm{lm}}$, but d, $\mathbf{d}^{\prime} \in \mathcal{D}_{\underline{w}_{0}}^{q}, \mathbf{d}^{\prime \prime} \notin \mathcal{D}^{q}$.

We show in the following example that $\mathcal{S}_{\mathrm{gm}}$ is in general a proper subset of $\mathcal{S}_{\mathrm{lm}}$.

ExAMPLE 5.3. Let $\mathfrak{g}$ be of type $\mathrm{A}_{\mathrm{n}}$ and let $\mathbf{d}$ be defined by $d_{\alpha_{i, j}}=2^{(n-1)-(j-i)}$. It is clear that $\mathbf{d} \in \mathcal{D}$. If $\alpha_{i, j}, \alpha_{k, l}, \alpha_{i, l}, \alpha_{k, j}$ are four different positive roots in $\Delta_{+}$such that $\alpha_{i, j}+\alpha_{k, l}=\alpha_{i, l}+\alpha_{k, j}$, the indices must satisfy $1 \leq i<k \leq l<j \leq n$. In this case, we have $d_{\alpha_{i, j}}+d_{\alpha_{k, l}}>d_{\alpha_{i, l}}+d_{\alpha_{k, j}}$. Hence, by Proposition 5.1, we have $\mathbf{d} \in \mathcal{S}_{\operatorname{lm}}$.

For arbitrary $1 \leq i \leq n$, let $P\left(\varpi_{i}\right)$ be the polytope obtained in [1], such that its lattice points $S\left(\varpi_{i}\right):=P\left(\varpi_{i}\right) \cap \mathbb{N}^{\Delta_{+}}$parameterizes a basis of $V\left(\varpi_{i}\right)$. Furthermore, by the choice of $\mathbf{d}$, we have

$$
S\left(\varpi_{i}\right)=\left\{\mathbf{s} \in \mathbb{N}^{\Delta_{+}} \mid f^{\mathbf{s}} \cdot v_{\varpi_{i}}^{\mathbf{d}} \neq 0 \text { in } V^{\mathbf{d}}\left(\varpi_{i}\right)\right\} .
$$

But in general, for $\lambda=m_{1} \varpi_{1}+\cdots+m_{n} \varpi_{n} \in \mathcal{P}_{+}$, the Minkowski sum of lattice points $S\left(\varpi_{1}\right)^{+m_{1}}+\cdots+S\left(\varpi_{n}\right)^{+m_{n}}$ may not parameterize a basis of $V(\lambda)$. For instance, let $\mathfrak{g}$ be of type $A_{4}$, we have [loc.cit]: $\#\left(S\left(\varpi_{1}\right)+S\left(\varpi_{2}\right)+S\left(\varpi_{3}\right)+S\left(\varpi_{4}\right)\right)=1023$ but $\operatorname{dim} V\left(\varpi_{1}+\varpi_{2}+\varpi_{3}+\varpi_{4}\right)=1024$. Hence, in general, $\mathbf{d} \notin \mathcal{S}_{\mathrm{gm}}$.

5.2. FFLV polytopes. We start with recalling the Dyck paths and FFLV polytopes [8], [9].

A sequence $\mathbf{b}=\left(\delta_{1}, \ldots, \delta_{r}\right)$ of positive roots is called a Dyck path of type $\mathrm{A}_{\mathrm{n}}$ if $\delta_{1}=\alpha_{i, i}$ and $\delta_{r}=\alpha_{j, j}$ for $i \leq j$ are simple roots, and if $\delta_{m}=\alpha_{p, q}$, then $\delta_{m+1}=\alpha_{p+1, q}$ or $\delta_{m+1}=\alpha_{p, q+1}$.

Let $A=\{1,2, \ldots, n, \overline{n-1}, \ldots, \overline{1}\}$ be the totally ordered index set $1<2<\ldots<$ $n<\overline{n-1}<\ldots<\overline{1}$. A symplectic Dyck path is a sequence $\mathbf{b}=\left(\delta_{1}, \ldots, \delta_{r}\right)$ of positive roots (of $\mathfrak{s p}_{2 n}$ ) such that the first root is a simple root, $\beta_{1}=\alpha_{i, i}$; the last root is either a simple root $\beta_{r}=\alpha_{j, j}$ or $\beta_{r}=\alpha_{j, \bar{j}}(i \leq j \leq n)$; if $\beta_{m}=\alpha_{r, q}$ with $r, q \in A$, then $\beta_{m+1}$ is either $\alpha_{r, q+1}$ or $\alpha_{r+1, q}$, where $x+1$ denotes the smallest element in $A$ which is bigger than $x$.

For a dominant integral weight $\lambda=\lambda_{1} \varpi_{1}+\lambda_{2} \varpi_{2}+\cdots+\lambda_{n} \varpi_{n}$ in the corresponding weight lattice, the FFLV polytopes $P_{\mathrm{A}_{\mathrm{n}}}(\lambda)$ and $P_{\mathrm{C}_{\mathrm{n}}}(\lambda)$ are defined by

$$
P_{\mathrm{A}_{\mathrm{n}}}(\lambda)=\left\{\mathbf{m} \in \mathbb{R}_{\geq 0}^{\Delta_{+}} \mid \begin{array}{c}
\text { for any } i=1, \ldots, n \text { and any Dyck paths } \mathbf{b}=\left(\delta_{1}, \ldots, \delta_{r}\right) \\
\text { starting in } \alpha_{i, i}, \text { ending in } \alpha_{j, j}: \sum_{\ell=1}^{r} m_{\delta_{\ell}} \leq \lambda_{i}+\cdots+\lambda_{j}
\end{array}\right\} ;
$$

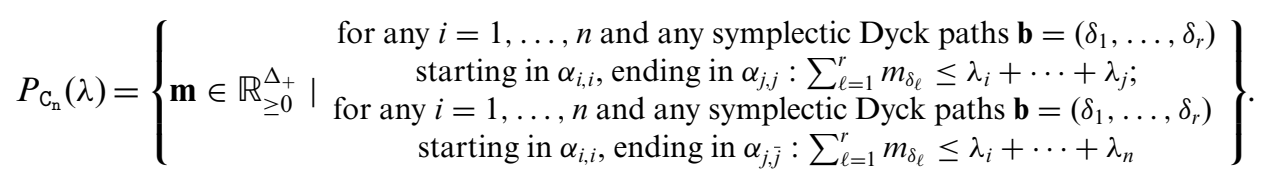


Let $S_{\mathrm{A}_{\mathrm{n}}}(\lambda)$ and $S_{\mathrm{C}_{\mathrm{n}}}(\lambda)$ denote the set of lattice points in the corresponding polytopes. It has been shown in [8] and [9] that the polytopes satisfy for all $\lambda=\lambda_{1}+\lambda_{2}$ :

$$
S_{\mathrm{A}_{\mathrm{n}}}(\lambda)=S_{\mathrm{A}_{\mathrm{n}}}\left(\lambda_{1}\right)+S_{\mathrm{A}_{\mathrm{n}}}\left(\lambda_{2}\right) \text { and } S_{\mathrm{C}_{\mathrm{n}}}(\lambda)=S_{\mathrm{C}_{\mathrm{n}}}\left(\lambda_{1}\right)+S_{\mathrm{C}_{\mathrm{n}}}\left(\lambda_{2}\right) .
$$

5.3. Global monomial sets: $A_{n}$. For $A_{n}$, consider $\mathbf{d} \in \mathcal{D}$ defined by $d_{\alpha_{i, j}}=(j-i+$ $1)(n-j+1)$, then the following theorem has been proved in [7]:

THEOREM 5.4.

(1) We have $\mathbf{d} \in \mathcal{S}_{\mathrm{gm}}$. Moreover, let $\underline{w}_{0}=\left(s_{n} \ldots s_{1}\right)\left(s_{n} \ldots s_{2}\right) \ldots\left(s_{n} s_{n-1}\right) s_{n}$, then $\mathbf{d} \in \mathcal{D}_{\underline{w}_{0}}^{q}$. (2) The set $\left\{f^{\mathbf{a}} \cdot v_{\lambda}^{\mathbf{d}} \mid \mathbf{a} \in S_{\mathrm{A}_{\mathrm{n}}}(\lambda)\right\}$ forms a monomial basis of $V^{\mathbf{d}}(\lambda)$.

5.4. Global monomial sets: $C_{n}$. Let us consider the $C_{n}$ case and $\mathbf{d} \in \mathcal{D}$ defined by

$$
d_{i, j}:=d_{\alpha_{i, j}}:=(2 n-j)(j-i+1), \quad d_{i, j}:=d_{\alpha_{i, j}}:=j(2 n-i-j+1) .
$$

This degree arises from an embedding of $\mathfrak{g}$ into a Lie algebra of type $A_{2 n-1}$. We will show that $\mathbf{d} \in \mathcal{S}_{\mathrm{gm}}$ and moreover, the monomial basis of $V^{\mathbf{d}}(\lambda)$ is parameterized by $S_{\mathrm{C}_{\mathrm{n}}}(\lambda)$. For this, we need an explicit description of the monomials associated to $S_{\mathrm{C}_{\mathrm{n}}}\left(\varpi_{k}\right)$ from [9]:

$$
\left\{f_{i_{1}, j_{\ell}-1} \cdots f_{i_{\ell}, j_{1}-1} \mid 1<i_{1}<\ldots<i_{\ell} \leq k \leq j_{1}<\ldots<j_{\ell}\right\}
$$

LEMma 5.5. The degree function $\mathbf{d} \in \mathcal{S}_{\operatorname{lm}}$ and for any fundamental weight $\varpi_{k}$,

$$
\left\{f^{\mathbf{a}} \cdot v_{\varpi_{k}}^{\mathbf{d}} \mid \mathbf{a} \in S_{\mathrm{C}_{\mathrm{n}}}\left(\varpi_{k}\right)\right\} \text { is a basis of } V^{\mathbf{d}}\left(\varpi_{k}\right) \text {. }
$$

Proof. We need to show that the annihilating ideal of $V^{\mathbf{d}}\left(\varpi_{k}\right)$ is monomial for all $\varpi_{k}$. We start with the natural representation, namely the vector space $\mathbb{C}^{2 n}$ with basis $\left\{e_{1}, \ldots, e_{n}, e_{\bar{n}}, \ldots, e_{\overline{1}}\right\}$ and operation $f_{i, j} e_{\ell}=\delta_{i, \ell} c_{i, j} e_{j+1}$ for some $c_{i, j} \in \mathbb{C}^{*}$ (when $j=\bar{p}$, we set $j+1:=\overline{p-1})$. We will further use that we can identify $V\left(\varpi_{k}\right)$ uniquely with a submodule in $\bigwedge^{k} \mathbb{C}^{2 n}$.

First of all, since $\mathbf{d} \in \mathcal{D}$, we see that we can restrict ourselves to the nilpotent radical of the fundamental weight $\varpi_{k}$ (since all other root vectors are acting by 0 on $v_{\varpi_{k}} \in V\left(\varpi_{k}\right)$ and hence on $\left.V^{\mathbf{d}}\left(\varpi_{k}\right)\right)$, e.g. we have to consider monomials in $M_{k}:=\left\{f_{i, j} \mid\right.$ $i \leq k \leq j\}$ only. We will prove the lemma in two steps:

(1) For any $i \leq k<j$, there exists a unique monomial $\mathbf{m}$ in the variables from $M_{k}$ with minimal degree such that $\mathbf{m} \cdot e_{i}=e_{j}$.

(2) For any $j_{1}<j_{2}<\ldots<j_{k}$ with $e_{j_{1}} \wedge \ldots \wedge e_{j_{k}} \in V\left(\varpi_{k}\right)$, there exists a unique monomial $\mathbf{m}$ in the variables from $M_{k}$ with minimal degree such that $\mathbf{m} \cdot e_{1} \wedge$ $e_{2} \wedge \ldots \wedge e_{k}=e_{j_{1}} \wedge e_{j_{2}} \wedge \ldots \wedge e_{j_{k}}$.

Then, the second step implies the Lemma.

We start with proving (1). Suppose $i \leq k \leq j \leq \bar{k}$, for weight reasons, there exists a unique monomial $\mathbf{m}$ in the variables from $M_{k}$ such that $\mathbf{m} \cdot e_{i}=e_{j}$, namely $\mathbf{m}=f_{\alpha_{i}, j-1}$. Suppose $i \leq k \leq n<\bar{k} \leq \bar{p}$, and for simplicity, we assume that $i \leq p$ (the $p \leq i$ case is 
similar). Let $\mathbf{m}$ be a monic monomial in the variables from $M_{k}$ such that $\mathbf{m} \cdot e_{i}=e_{\overline{p-1}}$, then for weight reasons $\mathbf{m}$ is in one of the following sets:

$$
\left\{f_{i, q-1} f_{p, \bar{q}}, f_{i, \bar{q}} f_{p, q-1} \mid q=k+1, \ldots, n\right\} \cup\left\{f_{i, n} f_{p, n}\right\} \cup\left\{f_{i, \bar{p}}\right\} .
$$

We will see that among these monomials, $f_{i, \bar{p}}$ is the unique monomial of minimal degree, namely of degree $p(2 n-i-p+1)$.

Let $i \leq q \leq n$ and denote

$$
\begin{aligned}
& \text { for } p<q, \quad Y(q):=q(2 n-i-q+1)+(2 n-(q-1))(q-p) \\
& \text { for } p \geq q, \quad Y(q):=q(2 n-i-q+1)+(2 n-p)(p-q+2) ; \\
& \text { for any } p, \quad X(q):=(2 n-(q-1))((q-1)-i+1)+q(2 n-p-q+1) .
\end{aligned}
$$

We have

$$
\begin{aligned}
& \text { if } p \leq q, \text { then } \operatorname{deg}_{\mathbf{d}}\left(f_{i, q-1} f_{p, \bar{q}}\right)=X(q) \\
& \text { if } p>q, \text { then } \operatorname{deg}_{\mathbf{d}}\left(f_{i, q-1} f_{q, \bar{p}}\right)=X(q) \\
& \text { if } p<q, \text { then } \operatorname{deg}_{\mathbf{d}}\left(f_{i, \bar{q}} f_{p, q-1}\right)=Y(q) \\
& \text { if } p \geq q \text {, then } \operatorname{deg}_{\mathbf{d}}\left(f_{i, \bar{q}} f_{q-1, p}\right)=Y(q) .
\end{aligned}
$$

Now, it is straightforward to see that for $q>i$ :

$$
X(q)>X(q-1) \text { and moreover } X(i)>p(2 n-i-p+1),
$$

as well as

$$
Y(q)>Y(q+1) \text { and } Y(n)=X(n) .
$$

Combining both gives

$$
Y(i)>\ldots>Y(n)=X(n)>\ldots>X(i)>p(2 n-i-p+1)=\operatorname{deg}_{\mathbf{d}} f_{i, \bar{p}} .
$$

Moreover,

$$
\operatorname{deg}_{\mathbf{d}}\left(f_{i, n} f_{p, n}\right)=n(2 n-i-p+2)>p(2 n-i-p+1)=p(2 n-i-p+1)=\operatorname{deg}_{\mathbf{d}} f_{i, \bar{p}} .
$$

This implies, that $f_{i, \bar{p}}$ is the unique monomial of minimal degree among all monomials in (9) and the first step is done.

We are left with step (2). Let $e_{i_{1}} \wedge \ldots \wedge e_{i_{k}} \in V\left(\varpi_{k}\right) \subset \wedge^{k} \mathbb{C}^{2 n}$, with $i_{1}<\ldots<i_{k}$ and $i_{j} \in\{1, \ldots, \overline{1}\}$. Let $\mathbf{m}$ be a monomial of minimal degree in the variables from $M_{k}$ such that

$$
\mathbf{m} \cdot e_{1} \wedge \ldots \wedge e_{k}=e_{i_{1}} \wedge \ldots \wedge e_{i_{k}}+\text { rest. }
$$

Due to the operation on the tensor product, there exists a factorization $\mathbf{m}=\prod_{\ell=1}^{k} \mathbf{m}_{\ell}$ and a permutation $\sigma \in \mathfrak{S}_{k}$, such that $\mathbf{m}_{\ell} \cdot e_{\ell}=e_{i_{\sigma(\ell)}}$. Since $\mathbf{m}$ is in the variables from $M_{k}$ only, we see that if $\ell \in\left\{i_{1}, \ldots, i_{k}\right\} \cap\{1, \ldots, k\}$, then $\mathbf{m}_{\ell}=1$ and hence $i_{\sigma}(\ell)=\ell$. So without loss of generality, we may assume that $k<i_{1}<i_{2}<\ldots<i_{k}$.

Suppose now there exist $\ell<j$ with $\sigma(\ell)<\sigma(j)$. We have

$$
\mathbf{m}_{\ell} \mathbf{m}_{j} \cdot e_{\ell} \wedge e_{j}=e_{i_{\sigma(\ell)}} \wedge e_{i_{\sigma(j)}}+\text { rest }
$$


From step (1), we deduce that if $\mathbf{m}$ is of minimal degree, then

$$
\mathbf{m}_{\ell}=f_{\ell, i_{\sigma(\ell)}-1}, \mathbf{m}_{j}=f_{j, i_{\sigma(j)}-1} .
$$

Similarly to the $A_{n}$ considerations (recall that the $C_{n}$-degree is a obtain from an $A_{2 n-1}$ degree), we see that

$$
\operatorname{deg}_{\mathbf{d}}\left(f_{\ell, i_{\sigma(j)}-1} f_{j, i_{\sigma(\ell)}-1}\right)<\operatorname{deg}_{\mathbf{d}}\left(f_{\ell, i_{\sigma(\ell)}-1} f_{j, i_{\sigma(j)}-1}\right) .
$$

We denote $\mathbf{m}^{\prime}:=f_{\ell, i_{\sigma())}-1} f_{j, i_{\sigma(\ell)}-1}\left(\prod_{i \neq j, \ell} \mathbf{m}_{i}\right)$, then

$$
\operatorname{deg}_{\mathbf{d}} \mathbf{m}>\operatorname{deg}_{\mathbf{d}} \mathbf{m}^{\prime} .
$$

But by construction,

$$
\mathbf{m}^{\prime} \cdot e_{1} \wedge \ldots \wedge e_{k}=e_{i_{1}} \wedge \ldots \wedge e_{i_{k}}+\text { rest }
$$

we have a contradiction to the minimality of the degree of $\mathbf{m}$ and hence $\sigma(\ell)>\sigma(j)$ for all $\ell<j$.

Let $\left\{i_{1} \ldots, i_{k}\right\}=\left\{p_{1}<\ldots<p_{s}\right\} \cup\left\{\ell_{1}<\ldots<\ell_{k-s}\right\}$, where $\ell_{k-s} \leq k<p_{1}$ and $\left\{q_{1}<\ldots<q_{s}\right\}$ be the complement of $\left\{\ell_{1}<\ldots<\ell_{k-s}\right\}$ in $\{1, \ldots, k\}$, then the monomial of minimal degree to obtain $e_{i_{1}} \wedge \ldots \wedge e_{i_{k}}$ is

$$
f_{q_{1}, p_{s}-1} \cdots f_{q_{s}, p_{1}-1} \text {. }
$$

This proves that $\mathbf{d} \in \mathcal{S}_{\mathrm{lm}}$ and moreover these are precisely the monomials associated to $S_{\mathrm{C}_{\mathrm{n}}}\left(\varpi_{k}\right)$.

From here, we can deduce by using (8) and Theorem 2.9:

THEOREM 5.6.

(1) For the degree function, $\mathbf{d} \in \mathcal{S}_{\mathrm{gm}}$.

(2) The set $\left\{f^{\mathbf{a}} \cdot v_{\lambda}^{\mathbf{d}} \mid \mathbf{a} \in S_{\mathrm{C}_{\mathrm{n}}}(\lambda)\right\}$ forms a monomial basis of $V^{\mathbf{d}}(\lambda)$.

(3) If $\mathbf{d} \in \mathcal{S}_{\mathrm{gm}}$ and the corresponding monomial basis is associated to $S_{\mathrm{C}_{\mathrm{n}}}(\lambda)$, then $\mathbf{d} \notin \mathcal{D}^{q}$.

Proof. Part (1) and (2) are deduced from the lemma, by using (8) and Theorem 2.9. It is left to prove the part (3), i.e. we assume that $\mathbf{d} \in \mathcal{D}$ satisfies (1) and (2) and we want to show $\mathbf{d} \notin \mathcal{D}^{q}$. We consider the simple Lie subalgebra $\mathfrak{g}_{2}$ of type $\mathrm{C}_{2}$ in $\mathfrak{g}$ with positive roots $\alpha_{n-1, n-1}, \alpha_{n-1, \overline{n-1}}, \alpha_{n-1, n}$ and $\alpha_{n, n}$. In the subalgebra $U_{q}\left(\mathfrak{g}_{2}\right) \subset U_{q}(\mathfrak{g})$, we have the following relation, independent of the chosen reduced expression (see Example 4.2),

$$
F_{n-1, \overline{n-1}} F_{n, n}=F_{n, n} F_{n-1, \overline{n-1}}+\left(1-q^{-2}\right) F_{n-1, n}^{(2)},
$$

implying that every $\mathbf{d}^{\prime} \in \mathcal{D}^{q}$ satisfies $\mathbf{d}_{n-1, \overline{n-1}}^{\prime}+\mathbf{d}_{n, n}^{\prime}>2 \mathbf{d}_{n-1, n}^{\prime}$.

Since d satisfies (1) and (2), in $V^{\mathbf{d}}\left(\varpi_{n}\right)$, we have $f_{n-1, n} f_{n, n} \cdot v_{\varpi_{n}}^{\mathbf{d}} \neq 0$ and $f_{n-1, n}^{2} \cdot v_{\varpi_{n}}^{\mathbf{d}}=$ 0 which implies $\mathbf{d}_{n-1, \overline{n-1}}+\mathbf{d}_{n, n}<2 \mathbf{d}_{n-1, n}$. Hence, $\mathbf{d} \notin \mathcal{D}^{q}$.

REMARK 5.7. If $\mathcal{S}_{\mathrm{gm}} \neq \emptyset$, then there exists an $\mathbb{N}$-filtration arising from $\mathbf{d} \in \mathcal{S}_{\mathrm{gm}}$ such that for any $\lambda \in \mathcal{P}_{+}, V^{\mathbf{d}}(\lambda)$ has a unique monomial basis.

If $\mathbf{d} \in \mathcal{D}_{\underline{w}_{0}}^{q} \cap \mathcal{S}_{\mathrm{gm}}$, then, by the argument in [7, Theorem 5], there exists an $\mathbb{N}$ filtration on $U_{q}\left(\mathfrak{n}^{-}\right)$arising from $\mathbf{d}$ such that for any $\lambda \in \mathcal{P}_{+}, V_{q}^{\mathbf{d}}(\lambda)$ has a unique monomial basis in $S_{q}\left(\mathfrak{n}^{-}\right)$. 
5.5. Global monomial set: $C_{2}$. Consider the quantum degree cone $\mathcal{D}_{\underline{w}_{0}}^{q}$ defined in (6). We pick a solution such that the sum $a_{1}+a_{2}+a_{3}+a_{4}$ takes its minimal value:

$$
\mathbf{d}=\left(d_{1,1}, d_{1, \overline{1}}, d_{1,2}, d_{2,2}\right)=(1,1,1,2) .
$$

Since $\mathbf{d} \in \mathcal{D}$, we consider the induced degree on the enveloping algebra with PBW root vectors $f_{1,1}, f_{1, \overline{1}}, f_{1,2}$ and $f_{2,2}$.

We turn to study whether $\mathbf{d}$ is in the global monomial set $\mathcal{S}_{\mathrm{gm}}$.

Let $\mathbf{S P}_{4}\left(m_{1}, m_{2}\right) \subset \mathbb{R}^{4}$ be the polytope defined by the following inequalities:

$$
\begin{gathered}
x_{1}, x_{2}, x_{3}, x_{4} \geq 0, x_{1} \leq m_{1}, x_{4} \leq m_{2}, \\
2 x_{1}+x_{2}+2 x_{3}+2 x_{4} \leq 2\left(m_{1}+m_{2}\right), x_{1}+x_{2}+x_{3}+2 x_{4} \leq m_{1}+2 m_{2} .
\end{gathered}
$$

Let $S\left(m_{1}, m_{2}\right)$ denote the lattice points in $\mathbf{S P}_{4}\left(m_{1}, m_{2}\right)$.

THEOREM 5.8. For any $\lambda=m_{1} \varpi_{1}+m_{2} \varpi_{2} \in \mathcal{P}_{+}$, the following statements hold:

(1) The set $\left\{f^{\mathbf{p}} v_{\lambda}^{\mathbf{d}} \mid \mathbf{p} \in S\left(m_{1}, m_{2}\right)\right\}$ forms a basis of $V^{\mathbf{d}}(\lambda)$, hence a basis of $V(\lambda)$.

(2) We have $\mathbf{d} \in \mathcal{S}_{\mathrm{gm}}$, i.e. the defining ideal $I^{\mathbf{d}}(\lambda)$ is monomial.

The proof that $\mathbf{d} \in \mathcal{S}_{\mathrm{lm}}$, can be deduced with the help of Corollary 2.8 (1). The rest of this paragraph will be devoted to prove this theorem.

Proposition 5.9. For any $m_{1}, m_{2}, m_{1}^{\prime}, m_{2}^{\prime} \in \mathbb{N}$,

$$
S\left(m_{1}, m_{2}\right)+S\left(m_{1}^{\prime}, m_{2}^{\prime}\right)=S\left(m_{1}+m_{1}^{\prime}, m_{2}+m_{2}^{\prime}\right) .
$$

Proof. It suffices to prove that for $m_{1}>0$ and $m_{2} \geq 0$,

$$
S\left(m_{1}-1, m_{2}\right)+S(1,0)=S\left(m_{1}, m_{2}\right) \text { and } S\left(0, m_{2}-1\right)+S(0,1)=S\left(0, m_{2}\right) .
$$

First, suppose that $m_{1} \neq 0$ and pick $\mathbf{s}=\left(a_{1}, a_{2}, a_{3}, a_{4}\right) \in S\left(m_{1}, m_{2}\right)$.

(1) If $a_{1} \neq 0$, we set $\mathbf{t}_{1}=\left(a_{1}-1, a_{2}, a_{3}, a_{4}\right)$ and $\mathbf{t}_{2}=(1,0,0,0)$; then $\mathbf{t}_{2} \in S(1,0)$. Since $\mathbf{s} \in S\left(m_{1}, m_{2}\right), 2 a_{1}+a_{2}+2 a_{3}+2 a_{4} \leq 2\left(m_{1}+m_{2}\right)$ implies that $2\left(a_{1}-1\right)+a_{2}+$ $2 a_{3}+2 a_{4} \leq 2\left(m_{1}-1+m_{2}\right) ; a_{1}+a_{2}+a_{3}+2 a_{4} \leq m_{1}+2 m_{2}$ implies that $a_{1}-1+$ $a_{2}+a_{3}+2 a_{4} \leq\left(m_{1}-1\right)+2 m_{2}$. Combining them together, we get $\mathbf{t}_{1} \in S\left(m_{1}-\right.$ $\left.1, m_{2}\right)$.

(2) If $a_{1}=0$ and $a_{3} \neq 0$, the very similar argument with $\mathbf{t}_{2}=(0,0,1,0)$ implies again $\mathbf{t}_{1}=\mathbf{s}-\mathbf{t}_{2} \in S\left(m_{1}-1, m_{2}\right)$.

(3) Suppose that $a_{1}=0, a_{3}=0$ but $a_{2} \neq 0$. The inequalities for $\mathbf{s} \in S\left(m_{1}, m_{2}\right)$ are reduced to $a_{2}+2 a_{4} \leq m_{1}+2 m_{2}$. We see that $\mathbf{s}=\left(0, a_{2}-1,0, a_{4}\right)+(0,1,0,0)$ gives a decomposition in $S\left(m_{1}-1, m_{2}\right)+S(1,0)$.

(4) When $a_{1}=a_{2}=a_{3}=0$ but $a_{4} \neq 0$, the decomposition is obvious.

Suppose now $m_{1}=0$ and pick $\mathbf{s}=\left(a_{1}, a_{2}, a_{3}, a_{4}\right) \in S\left(0, m_{2}\right)$. Then, $a_{1}=0$ and the inequality $a_{2}+a_{3}+2 a_{4} \leq 2 m_{2}$ is redundant.

(1) Suppose $a_{3} \neq 0$, then we decompose $\mathbf{s}=\left(0, a_{2}-1, a_{3}, a_{4}\right)+(0,1,0,0)$ : It is clear $(0,1,0,0) \in S(0,1)$; since $a_{2}+2 a_{3}+2 a_{4} \leq 2 m_{2}$, we get $a_{2}+2\left(a_{3}-1\right)+2 a_{4} \leq$ $2\left(m_{2}-1\right)$, it implies $\left(0, a_{2}-1, a_{3}, a_{4}\right) \in S\left(0, m_{2}-1\right)$.

(2) The case $a_{1}=a_{3}=0$ but $a_{4} \neq 0$ can be dealt in a similar way. 
(3) We are left with the case where $0 \neq a_{2} \leq 2 m_{2}$. If $a_{2} \leq 2$, there is nothing to be shown; if $a_{2}>2$, we decompose it as $\left(0, a_{2}-2,0,0\right)+(0,2,0,0)$.

Repeating this procedure shows that any element in $S\left(m_{1}, m_{2}\right)$ can be decomposed as the sum of elements in $S\left(m_{1}-k, m_{2}-\ell\right)$ and in $S(k, \ell)$.

To apply Theorem 2.9 to terminate the proof of Theorem 5.8, it suffices to count the number of lattice points in $\mathbf{S P}_{4}\left(m_{1}, m_{2}\right)$.

For any integers $a, b \in \mathbb{N}$, we define a polytope $\mathbf{P}(a, b) \subset \mathbb{R}^{2}$ by the following inequalities:

$$
x \geq 0, \quad y \geq 0, \quad x+2 y \leq a, \quad x+y \leq b .
$$

Let $N(a, b)$ denote the number of lattice points in $\mathbf{P}(a, b)$.

LEMMA 5.10. The number of lattice points $N(a, b)$ has the following expression:

(1) $N(a, a)=\left\{\begin{array}{l}l(l+1) \text { if } a=2 l-1 \\ (l+1)^{2} \quad \text { if } a=2 l .\end{array}\right.$

(2) $N(a, b)=\left\{\begin{array}{cc}N(a, a), & \text { if } b \geq a ; \\ \frac{1}{2}(b+1)(b+2), & \text { if } a \geq 2 b ; \\ -l^{2}+2 l b-\frac{1}{2} b^{2}+\frac{1}{2} b+l+1, & \text { if } 2 b>a>b \text { and } a=2 l ; \\ -l^{2}+2 l b-\frac{1}{2} b^{2}+\frac{3}{2} b+1, & \text { if } 2 b>a>b \text { and } a=2 l+1 .\end{array}\right.$

Proof. It amounts to count the integral points in the closed region cutting by the lines $x+2 y=a, x+y=b$ and the two axes in $\mathbb{R}^{2}$ which depends on the position of the intersection of these two lines.

Proposition 5.11. The number of lattice points in $\mathbf{S P}_{4}\left(m_{1}, m_{2}\right)$ is

$$
\frac{1}{6}\left(m_{1}+1\right)\left(m_{2}+1\right)\left(m_{1}+m_{2}+2\right)\left(m_{1}+2 m_{2}+3\right) .
$$

Proof. Let $H$ be the intersection of hyperplanes $x_{1}=\alpha$ and $x_{4}=\beta$ in $\mathbb{R}^{4}$ with coordinates $\left(x_{1}, x_{2}, x_{3}, x_{4}\right)$ where $\alpha, \beta \geq 0$. By definition,

$$
H \cap \mathbf{S P}_{4}\left(m_{1}, m_{2}\right)=\mathbf{P}\left(2 m_{1}+2 m_{2}-2 \alpha-2 \beta, m_{1}+2 m_{2}-\alpha-2 \beta\right) .
$$

Therefore, by Lemma 5.10, the number of integral points in $\mathbf{S P}_{4}\left(m_{1}, m_{2}\right)$ equals

$$
\sum_{\alpha=0}^{m_{1}} \sum_{\beta=0}^{m_{2}} N\left(2 m_{1}+2 m_{2}-2 \alpha-2 \beta, m_{1}+2 m_{2}-\alpha-2 \beta\right) .
$$

Since $\alpha \leq m_{1}$ and $\beta \leq m_{2}$, it falls into the third case in Lemma 5.10 (2) and (10) reads (where $l=m_{1}+m_{2}-\alpha-\beta$ and $b=m_{1}+2 m_{2}-\alpha-2 \beta$ ):

$$
\begin{aligned}
& \sum_{\alpha=0}^{m_{1}} \sum_{\beta=0}^{m_{2}} \frac{1}{2} \alpha^{2}+2 \alpha \beta+\beta^{2}-\left(m_{1}+2 m_{2}+\frac{3}{2}\right) \alpha-2\left(m_{1}+m_{2}+1\right) \beta \\
& \quad+\left(\frac{1}{2} m_{1}^{2}+2 m_{1} m_{2}+m_{2}^{2}+\frac{3}{2} m_{1}+2 m_{2}+1\right) .
\end{aligned}
$$

An easy summation provides the number in the statement. 
By Weyl character formula, for $\lambda=m_{1} \varpi_{1}+m_{2} \varpi_{2} \in \mathcal{P}_{+}, \operatorname{dim} V(\lambda)$ coincides with the number of lattice points in $\mathbf{S P}_{4}\left(m_{1}, m_{2}\right)$. This terminates the proof of Theorem 5.8.

REMARK 5.12. By permuting the second and the third coordinates, the polytope $\mathbf{S P}_{4}\left(m_{1}, m_{2}\right)$ coincides with the one in Proposition 4.1 of [15] (see also [16]), which is unimodularly equivalent to the Newton-Okounkov body of some valuation arising from inclusions of (translated) Schubert varieties.

There are several other known polytopes parameterizing bases of a finitedimensional irreducible representation $V(\lambda)$ of $\mathfrak{s p}_{4}$. For example, the Gelfand-Tstelin polytope $P_{1}(\lambda)$ [3]; the FFLV polytope $P_{2}(\lambda)$ [9]; the string polytope $P_{3}(\lambda)$ associated to the reduced decomposition $\underline{w}_{0}=s_{1} s_{2} s_{1} s_{2}$ [17]; the string polytope $P_{4}(\lambda)$ associated to the reduced decomposition $\underline{w}_{0}=s_{2} s_{1} s_{2} s_{1}$ [loc.cit.]; when $\lambda=m_{1} \varpi_{1}+m_{2} \varpi_{2}$, the polytope $\mathbf{S P}_{4}\left(m_{1}, m_{2}\right)$.

With the help of Polymake [11], one can verify that the polytopes $P_{1}(\lambda), P_{2}(\lambda)$ and $P_{4}(\lambda)$ are unimodular equivalent; but the polytope $P_{3}(\lambda)$ and $\mathbf{S P}_{4}\left(m_{1}, m_{2}\right)$ are not unimodular equivalent to any other polytopes.

REMARK 5.13. Using the polyhedral cones associated to these polytopes, the construction in [6] can be applied to produce three non-isomorphic toric degenerations of the spherical varieties associated to the symplectic group $\mathrm{Sp}_{4}$, see for example $[\mathbf{6}$, Sections 10, 12, 13].

5.6. Global monomial set: $D_{4}$. We prove that the global monomial set for $D_{4}$ is non-empty. We refer to Section 6.4 for details on the cones and the enumeration of positive roots. Let $\mathbf{d}=(5,5,1,2,4,1,1,2,6,10,12,20)$. It is shown in Section 6.4 that there exists a $\underline{w}_{0} \in R\left(w_{0}\right)$ such that $\mathbf{d} \in \mathcal{D}_{\underline{w}_{0}}^{q}$. We will freely use the notations in Section 6.4 .

Let $P_{\mathrm{D}_{4}}(\lambda)$ be the polytope defined in [13, Section 3] and $S_{\mathrm{D}_{4}}(\lambda)$ be the set of lattice points in $P_{\mathrm{D}_{4}}(\lambda)$.

\section{THEOREM 5.14.}

(1) We have $\mathbf{d} \in \mathcal{S}_{\mathrm{gm}}$.

(2) The set $\left\{f^{\mathbf{s}} \cdot v_{\lambda}^{\mathbf{d}} \mid \mathbf{s} \in S_{\mathrm{D}_{4}}(\lambda)\right\}$ forms a monomial basis of $V^{\mathbf{d}}(\lambda)$.

(3) Let $\underline{w}_{0}=s_{2} s_{1} s_{2} s_{3} s_{2} s_{4} s_{2} s_{1} s_{2} s_{3} s_{2} s_{4} \in R\left(w_{0}\right)$, then $\mathbf{d} \in \mathcal{D}_{\underline{w}_{0}}^{q}$.

Proof. The part of the proof that gives $\mathbf{d} \in \mathcal{S}_{\mathrm{lm}}$ is straightforward with Corollary 2.8. By a straightforward comparation, we obtain

$$
S_{\mathrm{D}_{4}}\left(\varpi_{i}\right)=\left\{\mathbf{s} \in \mathbb{N}^{\Delta_{+}} \mid f^{\mathbf{s}} \cdot v_{\varpi_{i}}^{\mathbf{d}} \neq 0 \text { in } V^{\mathbf{d}}\left(\varpi_{i}\right)\right\}, \quad i=1,2,3,4 .
$$

It is shown in [loc.cit.] that for any $\lambda, \mu \in \mathcal{P}_{+}$, we have

$$
P_{\mathrm{D}_{4}}(\lambda)+P_{\mathrm{D}_{4}}(\mu)=P_{\mathrm{D}_{4}}(\lambda+\mu) \text { and } S_{\mathrm{D}_{4}}(\lambda)+S_{\mathrm{D}_{4}}(\mu)=S_{\mathrm{D}_{4}}(\lambda+\mu)
$$

and $\operatorname{dim} V(\lambda)=\# S_{\mathrm{D}_{4}}(\lambda)$.

The statements (1) and (2) follow from Theorem 2.9. The part (3) is shown in Section 6.4 .

5.7. Global monomial set: $B_{3}$. Let $\mathfrak{g}$ be of type $B_{3}$. For $\lambda \in \mathcal{P}_{+}$, let $P_{B_{3}}(\lambda)$ denote the polytope defined in [2, Section 5] and $S_{\mathrm{B}_{3}}(\lambda)$ be the set of lattice points in $P_{\mathrm{B}_{3}}(\lambda)$. 
Let $f_{i, j}$ and $f_{i, \bar{j}}$ be the PBW root vectors associated to the positive roots $\alpha_{i, j}$ and $\alpha_{i, \bar{j}}$, respectively. For $\mathbf{d} \in \mathbb{R}_{\geq 0}^{\Delta_{+}}$, we write $d_{i, j}=\mathbf{d}\left(\alpha_{i, j}\right)$ and $d_{i, \bar{j}}=\mathbf{d}\left(\alpha_{i, \bar{j}}\right)$. We consider the element $\mathbf{d} \in \mathbb{R}_{\geq 0}^{\Delta_{+}}$defined by

$d_{1,1}=4, d_{1,2}=3, d_{2,2}=3, d_{1,3}=3, d_{1, \overline{2}}=1, d_{1, \overline{3}}=1, d_{2,3}=4, d_{2, \overline{3}}=3, d_{3,3}=2$.

We will show in Section 6.2 that $\mathbf{d} \in \mathcal{D}$.

THEOREM 5.15 .

(1) We have $\mathbf{d} \in \mathcal{S}_{\mathrm{gm}}$, and the set $\left\{f^{\mathbf{s}} \cdot v_{\lambda}^{\mathbf{d}} \mid \mathbf{s} \in S_{\mathrm{B}_{3}}(\lambda)\right\}$ forms a monomial basis of $V^{\mathbf{d}}(\lambda)$.

(2) For any $\mathbf{e} \in \mathcal{D}$ satisfying (1), we have $\mathbf{e} \notin \mathcal{D}^{q}$.

Proof.

(1) As before by computing each weight space in $V^{\mathbf{d}}\left(\varpi_{i}\right), i=1,2,3$, we obtain $\mathbf{d} \in \mathcal{S}_{\operatorname{lm}}$. By comparing the basis arising from the monomiality of the defining ideals $I^{\mathbf{d}}\left(\varpi_{i}\right)$ with the basis obtain in [loc.cit.], we get for $i=1,2,3$ :

$$
S_{\mathrm{B}_{3}}\left(\varpi_{i}\right)=\left\{\mathbf{s} \in \mathbb{N}^{\Delta_{+}} \mid f^{\mathbf{s}} \cdot v_{\varpi_{i}}^{\mathbf{d}} \neq 0 \text { in } V^{\mathbf{d}}\left(\varpi_{i}\right)\right\} .
$$

For any $\lambda, \mu \in \mathcal{P}_{+}$, we have

$$
P_{\mathrm{B}_{3}}(\lambda)+P_{\mathrm{B}_{3}}(\mu)=P_{\mathrm{B}_{3}}(\lambda+\mu), \quad S_{\mathrm{B}_{3}}(\lambda)+S_{\mathrm{B}_{3}}(\mu)=S_{\mathrm{B}_{3}}(\lambda+\mu)
$$

and $\operatorname{dim} V(\lambda)=\# S_{\mathrm{B}_{3}}(\lambda)$. By Theorem 2.9, $\mathbf{d} \in \mathcal{S}_{\mathrm{gm}}$.

(2) Let $\mathbf{e} \in \mathcal{S}_{\mathrm{gm}}$. From reading the lattice points in $P_{\mathrm{B}_{3}}\left(\varpi_{2}\right)$, we get $f_{1,2} f_{1, \overline{3}} \cdot v_{\varpi_{2}}^{\mathbf{e}} \neq 0$ in $V^{\mathbf{e}}\left(\varpi_{2}\right)$. Since the corresponding weight space is one-dimensional and $f_{1,3}^{2}$ has the same weight, $f_{1,3}^{2} \cdot v_{\varpi_{2}}^{\mathbf{e}}=0$.

Assume $\underline{w}_{0} \in R\left(w_{0}\right)$ such that $\mathbf{e} \in \mathcal{D}_{\underline{w}_{0}}^{q}$. Let $<$ be the induced convex order on $\Delta_{+}$. Without loss of generality, we suppose that $\alpha_{1,2}<\alpha_{1, \overline{3}}$.

CASE 1. Assume $\alpha_{1,2}<\alpha_{1,3}<\alpha_{1, \overline{3}}$, for the quantum degree cone $\mathcal{D}_{\underline{w}_{0}}^{q}$, by computing the $\mathrm{L}-\mathrm{S}$ formula explicitly, this would imply the following inequality: $d_{1,2}+d_{1, \overline{3}}>2 d_{1,3}$. This implies, turning to the classical case, $f_{1,3}^{2} \cdot v_{\varpi_{2}}^{\mathbf{e}} \neq 0$, which is a contradiction.

CAse 2. Assume $\alpha_{1,3}<\alpha_{1,2}<\alpha_{1, \overline{3}}$. Consider the root $\alpha_{3,3}$ : By the convexity, it must be simultaneously larger than $\alpha_{1, \overline{3}}$ and smaller than $\alpha_{1,2}$. This is a contradiction.

CASE 3. Assume $\alpha_{1,2}<\alpha_{1, \overline{3}}<\alpha_{1,3}$, with similar arguments as in Case 2 we get a contradiction.

As a conclusion, for any $\underline{w}_{0} \in R\left(w_{0}\right), \mathbf{e} \notin \mathcal{D}_{\underline{w}_{0}}^{q}$.

5.8. Global monomial set: $G_{2}$. Let $\mathfrak{g}$ be of type $G_{2}$. We use the notations in Section 4.3. Consider the following $\mathbf{d} \in \mathcal{D}$ :

$$
d_{1}=2, \quad d_{1112}=1, \quad d_{112}=3, \quad d_{11122}=1, \quad d_{12}=3, \quad d_{2}=2 .
$$

It is clear that $\mathbf{d} \in \mathcal{D}$.

Let $P_{\mathrm{G}_{2}}(\lambda)$ be the polytope defined in [12, Section 1], the set of its lattice points will be denoted by $S_{\mathrm{G}_{2}}(\lambda)$. With similar arguments and calculations as before, we 
obtain the first statement of the following theorem. The second statement follows from Section 4.3, there we show, for each $\mathbf{e} \in \mathcal{D}^{q}$, there exist a unique monomial basis of $V^{\mathrm{e}}\left(\varpi_{i}\right), i=1,2$, i.e. $\mathbf{e} \in \mathcal{S}_{\mathrm{lm}}$, which does not coincide with the basis of the following theorem.

THEOREM 5.16. We have $\mathbf{d} \in \mathcal{S}_{\mathrm{gm}}$, and the set $\left\{f^{\mathbf{s}} \cdot v_{\lambda}^{\mathbf{d}} \mid \mathbf{s} \in S_{\mathrm{G}_{2}}(\lambda)\right\}$ forms a monomial basis of $V^{\mathbf{d}}(\lambda)$.

REMARK 5.17. There is no $\mathbf{d} \in \mathcal{D}^{q}$, such that $\left\{f^{\mathbf{s}} \cdot v_{\lambda}^{\mathbf{d}} \mid \mathbf{s} \in S_{\mathrm{G}_{2}}(\lambda)\right\}$ is a basis of $V^{\mathbf{d}}(\lambda)$.

5.9. Local monomial sets: $G_{2}$. Let $\mathfrak{g}$ be of type $G_{2}$. By Proposition 4.4 , the quantum degree cone $\mathcal{D}_{\underline{w}_{0}}^{q}$ does not depend on the choice of $\underline{w}_{0} \in R\left(w_{0}\right)$. Let

$$
\mathbf{d}=\left(d_{1}, d_{1112}, d_{112}, d_{11122}, d_{12}, d_{2}\right)=(2,2,1,2,2,5) .
$$

We will show in Section 4.3 that $\mathbf{d} \in \mathcal{D}_{\underline{w}_{0}}^{q}$. Let $f_{1}, f_{1112}, f_{112}, f_{11122}, f_{12}, f_{2}$ be the PBW root vectors in $\mathfrak{n}^{-}$.

Proposition 5.18. We have $\mathbf{d} \in \mathcal{S}_{\operatorname{lm}}$, i.e. the defining ideals $I^{\mathbf{d}}\left(\varpi_{1}\right)$ and $I^{\mathbf{d}}\left(\varpi_{2}\right)$ are monomial.

We omit the proof as before.

We want to examine that this degree function is quite interesting, due to the fact that the induced semigroup is not saturated as we will explain.

Let $S\left(\varpi_{2}\right)=\left\{\mathbf{s} \in \mathbb{N}^{\Delta_{+}} \mid f^{\mathbf{s}} \cdot v_{\varpi_{2}}^{\mathbf{d}} \neq 0\right\}$. We have by construction $\# S\left(\varpi_{2}\right)=$ $\operatorname{dim} V\left(\varpi_{2}\right)=14$, but there are 16 lattice points in the convex hull $P=\operatorname{conv}\left(S\left(\varpi_{2}\right)\right)$.

We fix the sequence of positive roots $\left(\alpha_{1}, \alpha_{1112}, \alpha_{112}, \alpha_{11122}, \alpha_{12}, \alpha_{2}\right)$ to identify $\mathbb{R}^{\Delta_{+}}$ and $\mathbb{R}^{6}$. Let $\mathbf{G}_{2}^{\sigma_{1}}\left(m_{1}\right) \subset \mathbb{R}^{6}$ be the polytope defined by the inequalities:

$$
x_{1}, x_{2}, x_{3}, x_{4}, x_{5}, x_{6} \geq 0, x_{1} \leq m_{1}, x_{6} \leq 0,2 x_{1}+2 x_{2}+x_{3}+2 x_{4}+2 x_{5} \leq 2 m_{1} \text {. }
$$

Let $\mathbf{G}_{\mathbf{2}}^{\varpi_{2}}\left(m_{2}\right) \subset \mathbb{R}^{6}$ be the polytope defined by the inequalities:

$$
x_{1}, x_{2}, x_{3}, x_{4}, x_{5}, x_{6} \geq 0, x_{1} \leq 0,2 x_{2}+x_{3}+x_{4}+x_{5}+2 x_{6} \leq 2 m_{2} .
$$

Let $\left\{e_{1}, e_{2}, \ldots, e_{6}\right\}$ be the standard basis of $\mathbb{R}^{6}$.

Let $\lambda=m_{1} \varpi_{1}+m_{2} \varpi_{2} \in \mathcal{P}_{+}$. We conjecture that the number of lattice points in the Minkowski sum

$$
m_{1} \mathbf{G}_{\mathbf{2}}^{\varpi_{1}}(1)+m_{2}\left(\mathbf{G}_{\mathbf{2}}^{\varpi_{2}}(1) \cup\left\{3 e_{3}, 3 e_{5}\right\}\right)
$$

coincides with $\operatorname{dim} V\left(m_{1} \varpi_{1}+m_{2} \varpi_{2}\right)$.

REMARK 5.19. Note that the proof of Lemma 5.18 does not depend on the choice of $\mathbf{d} \in \mathcal{D}_{\underline{w}_{0}}^{q}$. Further we have $\mathcal{D}^{q}=\mathcal{D}_{\underline{w}_{0}}^{q}$ (see Proposition 4.4). This implies the inclusion $\mathcal{D}^{q} \subset \mathcal{S}_{\mathrm{lm}}$.

REMARK 5.20. Let $G$ be the complex algebraic group of type $\mathrm{G}_{2}$ and $U$ (resp. $U^{-}$) be the maximal unipotent subgroup of $G$ having $\mathfrak{n}^{+}$(resp. $\left.\mathfrak{n}^{-}\right)$as Lie algebra.

Let $S=\left(\alpha_{1}, \alpha_{1112}, \alpha_{112}, \alpha_{11122}, \alpha_{12}, \alpha_{2}\right)$ be a birational sequence for $U^{-}$(see [6, Section 3] for definition). Using $S$, we identify $\mathbb{N}^{\Delta_{+}}$and $\mathbb{N}^{6}$. We fix the integral weight function $\Psi: \Delta_{+} \rightarrow \mathbb{N}$ by $\Psi=\mathbf{d}$ and the lexicographic order on $\mathbb{N}^{6}$. Let $>$ be the total order on $\mathbb{N}^{6}$ defined in $[6$, Section 5] by combining $\Psi$ and the lexicographic 
order. In [loc.cit], a monoid $\Gamma=\Gamma(S,>) \subset \mathcal{P}_{\mathbb{R}} \times \mathbb{N}^{6}$ is attached to $G / / U$ to study its toric degenerations. Let $\pi_{1}: \mathcal{P}_{\mathbb{R}} \times \mathbb{N}^{6} \rightarrow \mathcal{P}_{\mathbb{R}}$ and $\pi_{2}: \mathcal{P}_{\mathbb{R}} \times \mathbb{N}^{6} \rightarrow \mathbb{N}^{6}$ be the canonical projections.

We claim that $\Gamma$ is not saturated: First notice that $\pi_{2} \circ \pi_{1}^{-1}\left(\varpi_{2}\right)=S\left(\varpi_{2}\right)$. Pick a lattice point $\mathbf{q} \in P$ which is not in $S\left(\varpi_{2}\right)$. Since $q \in \operatorname{conv}\left(S\left(\varpi_{2}\right)\right)$, there exists $s_{1}, \ldots, s_{m} \in \mathbb{Q}$ and $\mathbf{p}_{1}, \ldots, \mathbf{p}_{m} \in S\left(\varpi_{2}\right)$ such that $s_{1}+\cdots+s_{m}=1$ and $\mathbf{q}=$ $s_{1} \mathbf{p}_{1}+\cdots+s_{m} \mathbf{p}_{m}$. Multiplying both sides by the least common multiple $M$ of the denominators of $s_{1}, \ldots, s_{m}$, we know that $\left(M \varpi_{2}, M \mathbf{q}\right) \in \Gamma$ as $\Gamma$ is a monoid. If $\Gamma$ were saturated, $\left(M \varpi_{2}, M \mathbf{q}\right) \in \Gamma$ will imply $\left(\varpi_{2}, \mathbf{q}\right) \in \Gamma$, contradicts to $\pi_{2} \circ \pi_{1}^{-1}\left(\varpi_{2}\right)=$ $S\left(\varpi_{2}\right)$.

This example explains that the saturated assumption in [6] is necessary. We thank Peter Littelmann for pointing out this application.

\section{Higher rank examples of quantum degree cones.}

6.1. Lie algebra $A_{3}$. Let $\mathfrak{g}$ be of type $A_{3}$. For $\mathbf{d} \in \mathbb{R}_{\geq 0}^{\Delta_{+}}$, we write $d_{i, j}=\mathbf{d}\left(\alpha_{i, j}\right)$. The classical degree cone $\mathcal{D} \subset \mathbb{R}_{\geq 0}^{\Delta_{+}}$is defined by the following inequalities:

$$
d_{1,1}+d_{2,2}>d_{1,2}, \quad d_{2,2}+d_{3,3}>d_{2,3}, \quad d_{1,1}+d_{2,3}>d_{1,3}, \quad d_{1,2}+d_{3,3}>d_{1,3},
$$

Let $\underline{w}_{0}^{1}=s_{1} s_{2} s_{1} s_{3} s_{2} s_{1}$ and $\underline{w}_{0}^{2}=s_{1} s_{3} s_{2} s_{3} s_{1} s_{2} \in R\left(w_{0}\right)$. We claim that the corresponding quantum degree cones satisfy $\mathcal{D}_{\underline{w}_{0}^{1}}^{q} \cap \mathcal{D}_{\underline{w}_{0}^{2}}^{q}=\emptyset$.

Let $F_{i, j}\left(\right.$ resp. $F_{i, j}^{\prime}$ ) denote the quantum PBW root vector associated to $\underline{w}_{0}^{1}$ (resp. $\left.w_{0}^{2}\right)$ and root $\alpha_{i, j}$. We have the following commutation relations between the quantum PBW root vectors:

$$
F_{1,2} F_{2,3}=F_{2,3} F_{1,2}+\left(q-q^{-1}\right) F_{2,2} F_{1,3}, \quad F_{1,3}^{\prime} F_{2,2}^{\prime}=F_{2,2}^{\prime} F_{1,3}^{\prime}+\left(q-q^{-1}\right) F_{1,2}^{\prime} F_{2,3}^{\prime},
$$

giving two contradicting inequalities in the corresponding quantum degree cones:

$$
d_{1,2}+d_{2,3}>d_{2,2}+d_{1,3}, \quad d_{1,3}+d_{2,2}>d_{1,2}+d_{2,3} .
$$

Projecting to the corresponding coordinates proves the claim.

6.2. Lie algebra $B_{3}$. Let $\mathfrak{g}$ be of type $B_{3}$. The set of positive roots

$$
\Delta_{+}=\left\{\alpha_{1,1}, \alpha_{1,2}, \alpha_{1,3}, \alpha_{2,2}, \alpha_{2,3}, \alpha_{3,3}, \alpha_{1, \overline{3}}, \alpha_{2, \overline{3}}, \alpha_{1, \overline{2}}\right\} .
$$

For $\mathbf{d} \in \mathbb{R}_{\geq 0}^{\Delta_{+}}$, we write $d_{i, j}=\mathbf{d}\left(\alpha_{i, j}\right)$ and $d_{i, \bar{j}}=\mathbf{d}\left(\alpha_{i, j}\right)$. The classical degree cone $\mathcal{D}$ is determined by

$$
\begin{gathered}
d_{1,1}+d_{2,2}>d_{1,2}, \quad d_{1,1}+d_{2,3}>d_{1,3}, \quad d_{1,1}+d_{2, \overline{3}}>d_{1, \overline{3}}, \quad d_{1,2}+d_{3,3}>d_{1,3}, \\
d_{1,2}+d_{2, \overline{3}}>d_{1, \overline{2}}, \quad d_{2,2}+d_{3,3}>d_{2,3}, \quad d_{2,2}+d_{1, \overline{3}}>d_{1, \overline{2}}, \\
d_{1,3}+d_{2,3}>d_{1, \overline{2}}, \quad d_{1,3}+d_{3,3}>d_{1, \overline{3}}, \quad d_{2,3}+d_{3,3}>d_{2, \overline{3}} .
\end{gathered}
$$

For example, $\mathbf{d}=(4,3,3,3,1,1,4,3,2) \in \mathcal{D}$. 
We consider

$$
\underline{w}_{0}^{1}=s_{1} s_{2} s_{1} s_{3} s_{2} s_{1} s_{3} s_{2} s_{3} \text { and } \underline{w}_{0}^{2}=s_{1} s_{3} s_{2} s_{3} s_{2} s_{1} s_{2} s_{3} s_{2} \in R\left(w_{0}\right) .
$$

The quantum degree cone $\mathcal{D}_{\underline{w}_{0}^{1}}^{q}$ in $\mathcal{D}$ is defined by the following inequalities:

$$
\begin{aligned}
& d_{1,1}+d_{1, \overline{2}}>2 d_{1,3}, \quad d_{1,2}+d_{1, \overline{2}}>d_{2,2}+2 d_{1,3}, \quad d_{1,2}+d_{1, \overline{3}}>2 d_{1,3}, \quad d_{1,2}+d_{2, \overline{3}} \\
& >d_{2,2}+d_{1, \overline{3}} \text {, } \\
& d_{1,2}+d_{2, \overline{3}}>d_{1,3}+d_{1,2}, \quad d_{2,2}+d_{2, \overline{3}}>2 d_{2,3}, \quad d_{1,3}+d_{2, \overline{3}}>d_{1, \overline{3}}+d_{2,3}, \\
& d_{1, \overline{2}}+d_{2, \overline{3}}>d_{1, \overline{3}}+2 d_{2,3}, \quad d_{1, \overline{2}}+d_{3,3}>d_{1, \overline{3}}+d_{2,3}, \quad d_{1,2}+d_{2,3}>d_{1,3}+d_{2,2} .
\end{aligned}
$$

The quantum degree cone $\mathcal{D}_{\underline{w}_{0}^{2}}^{q}$ in $\mathcal{D}$ is defined by the following inequalities:

$$
\begin{gathered}
d_{1,1}+d_{1, \overline{2}}>2 d_{1,3}, \quad d_{1,1}+d_{1, \overline{2}}>d_{1,2}+d_{1, \overline{3}}, \quad d_{1,2}+d_{1, \overline{3}}>2 d_{1,3}, \quad d_{1, \overline{3}}+d_{2,3} \\
>d_{1,3}+d_{2, \overline{3}}, \\
d_{1, \overline{3}}+d_{2,2}>d_{1,3}+d_{2,3}, \quad d_{1, \overline{3}}+d_{2,2}>d_{1,2}+d_{2, \overline{3}}, \quad d_{1,3}+d_{2,3}>d_{1,2}+d_{2, \overline{3}}, \\
d_{2, \overline{3}}+d_{2,2}>2 d_{2,3}, \quad d_{1,3}+d_{2,2}>d_{1,2}+d_{2,3} .
\end{gathered}
$$

By the contradiction of the last inequalities, we obtain $\mathcal{D}_{\underline{w}_{0}^{1}}^{q} \cap \mathcal{D}_{\underline{w}_{0}^{2}}^{q}=\emptyset$.

6.3. Lie algebra $C_{3}$. Let $\mathfrak{g}$ be of type $C_{3}$. For $\mathbf{d} \in \mathbb{R}_{\geq 0}^{\Delta_{+}}$, we write $d_{1}=\mathbf{d}\left(\alpha_{1,1}\right), d_{2}=$ $\mathbf{d}\left(\alpha_{1,2}\right), d_{3}=\mathbf{d}\left(\alpha_{1, \overline{1}}\right), d_{4}=\mathbf{d}\left(\alpha_{1,3}\right), d_{5}=\mathbf{d}\left(\alpha_{1, \overline{2}}\right), d_{6}=\mathbf{d}\left(\alpha_{2,2}\right), d_{7}=\mathbf{d}\left(\alpha_{2, \overline{2}}\right), d_{8}=\mathbf{d}\left(\alpha_{2,3}\right)$ and $d_{9}=\mathbf{d}\left(\alpha_{3,3}\right)$. The classical degree cone $\mathcal{D} \subset \mathbb{R}_{\geq 0}^{\Delta_{+}}$is determined by

$$
\begin{aligned}
& d_{1}+d_{5}>d_{3}, \quad d_{1}+d_{6}>d_{2}, \quad d_{1}+d_{7}>d_{5}, \quad d_{1}+d_{8}>d_{4}, \quad d_{2}+d_{4}>d_{3}, \\
& d_{2}+d_{8}>d_{5}, \quad d_{2}+d_{9}>d_{4}, \quad d_{4}+d_{6}>d_{5}, \quad d_{6}+d_{8}>d_{7}, \quad d_{6}+d_{9}>d_{8} .
\end{aligned}
$$

We consider the reduced decompositions

$$
\underline{w}_{0}^{1}=s_{1} s_{2} s_{3} s_{2} s_{1} s_{2} s_{3} s_{2} s_{3} \text { and } \underline{w}_{0}^{2}=s_{1} s_{3} s_{2} s_{3} s_{2} s_{1} s_{2} s_{3} s_{2} .
$$

Moreover, the inequalities determining the cone $\mathcal{D}_{\underline{w}_{0}^{1}}^{q}$ in $\mathcal{D}$ are

$$
\begin{gathered}
d_{1}+d_{5}>d_{2}+d_{4}, \quad d_{3}+d_{9}>2 d_{4}, \quad d_{7}+d_{9}>2 d_{8}, \quad d_{3}+d_{7}>2 d_{5}, \\
d_{1}+d_{7}>d_{4}+d_{6}, \quad d_{2}+d_{7}>d_{5}+d_{6}, \quad d_{2}+d_{7}>d_{4}+2 d_{6}, \quad d_{3}+d_{7}>d_{4}+d_{5}+d_{6}, \\
d_{3}+d_{7}>2 d_{4}+2 d_{6}, \quad d_{3}+d_{8}>d_{4}+d_{5}, \quad d_{3}+d_{8}>2 d_{4}+d_{6}, \quad d_{2}+d_{8}>d_{4}+d_{6} .
\end{gathered}
$$

The inequalities determining the cone $\mathcal{D}_{\underline{w}_{0}^{2}}^{q}$ in $\mathcal{D}$ are

$$
\begin{gathered}
d_{1}+d_{5}>d_{2}+d_{4}, \quad d_{3}+d_{9}>2 d_{4}, \quad d_{7}+d_{9}>2 d_{8}, \quad d_{3}+d_{7}>2 d_{5}, \\
d_{1}+d_{7}>d_{2}+d_{8}, \quad d_{4}+d_{7}>d_{5}+d_{8}, \quad d_{3}+d_{7}>d_{2}+d_{5}+d_{8}, \quad d_{3}+d_{7}>2 d_{2}+2 d_{8}, \\
d_{3}+d_{6}>d_{2}+d_{5}, \quad d_{3}+d_{6}>2 d_{2}+d_{8}, \quad d_{4}+d_{7}>d_{2}+d_{8}, \quad d_{4}+d_{6}>d_{2}+d_{8} .
\end{gathered}
$$

Notice that there is a contradiction in the last inequalities, implying that $\mathcal{D}_{\underline{w}_{0}^{1}}^{q} \cap \mathcal{D}_{\underline{w}_{0}^{2}}^{q}=\emptyset$. 

entries:

There are four elements in $\mathcal{D}_{\underline{w}_{0}^{1}}^{q}$, which are minimal regarding the sum over all

$$
\begin{aligned}
& \mathbf{d}_{1}=(2,1,1,1,1,1,4,4,5), \mathbf{d}_{2}=(3,2,2,1,1,1,3,3,4), \\
& \mathbf{d}_{3}=(5,4,4,1,1,1,1,1,2), \mathbf{d}_{4}=(4,3,3,1,1,1,2,2,3) .
\end{aligned}
$$

Since $\mathbf{d}_{1}, \mathbf{d}_{2}, \mathbf{d}_{3}, \mathbf{d}_{4} \in \mathcal{D}$, we go back to the classical case. We consider the fundamental module $V\left(\varpi_{2}\right)$ and the weight $\tau=2 \alpha_{1}+3 \alpha_{2}+\alpha_{3}$ whose weight space $V\left(\varpi_{2}\right)_{\varpi_{2}-\tau}$ is of dimension 1 . We have to choose an element with minimal degree from the following set, where we neglect the elements which have obviously a higher degree:

$$
\left\{f_{1,2} f_{1, \overline{2}}, f_{1, \overline{1}} f_{2,2}\right\} \text {. }
$$

For each of the above elements in $\mathcal{D}_{\underline{w}_{0}}^{q}$, both monomials have the same degree, so we do not obtain a monomial ideal $I^{\mathbf{d}_{i}}, 1 \leq i \leq 4$.

By taking larger degrees $\mathbf{d} \in \mathcal{D}_{\underline{w}_{0}}^{q}$, it is possible to obtain a unique monomial basis of $V^{\mathbf{d}}\left(\varpi_{2}\right)$, where it is possible to obtain a basis with either of both monomials applied to $v_{\varpi_{2}}^{\mathbf{d}}$. We conclude $\mathcal{D}_{\underline{w}_{0}}^{q} \nsubseteq \mathcal{S}_{\mathrm{lm}}$, but $\mathcal{D}_{\underline{w}_{0}}^{q} \cap \mathcal{S}_{\mathrm{lm}} \neq \emptyset$. We also see, different elements in $\mathcal{D}_{\underline{w}_{0}}^{q}$ can produce different monomial bases. This observation still holds, even if we consider elements where the sum over the entries is the same.

6.4. Lie algebra $D_{4}$. Let $\mathfrak{g}$ be of type $D_{4}$. In the Dynkin diagram, we let 2 be the central node. We consider the following reduced decomposition

$$
\underline{w}_{0}=s_{2} s_{1} s_{2} s_{3} s_{2} s_{4} s_{2} s_{1} s_{2} s_{3} s_{2} s_{4} \in R\left(w_{0}\right) .
$$

For a positive root $a \alpha_{1}+b \alpha_{2}+c \alpha_{3}+d \alpha_{4} \in \Delta_{+}$, we let $f_{a b c d}$ denote the corresponding quantum $\mathrm{PBW}$ root vector. In the convex order on positive roots given by $\underline{w}_{0}$, they are

$f_{0100}, f_{1100}, f_{1000}, f_{1110}, f_{0110}, f_{1211}, f_{1101}, f_{1111}, f_{0010}, f_{0111}, f_{0101}, f_{0001}$.

For $d \in \mathbb{R}_{\geq 0}^{\Delta_{+}}$, let $d_{i}$ be the value of $\mathbf{d}$ at the positive root corresponding to the $i$ th quantum PBW root vector above. The quantum degree cone $\mathcal{D}_{\underline{w}_{0}}^{q} \subset \mathbb{R}_{\geq 0}^{\Delta_{+}}$is defined by

$$
\begin{gathered}
d_{1}+d_{3}>d_{2}, \quad d_{1}+d_{8}>d_{5}+d_{7}, \quad d_{1}+d_{8}>d_{6}, \quad d_{1}+d_{9}>d_{5}, \quad d_{1}+d_{12}>d_{11} \\
d_{2}+d_{8}>d_{3}+d_{5}+d_{7}, \quad d_{2}+d_{8}>d_{3}+d_{6}, \quad d_{2}+d_{8}>d_{4}+d_{7}, \quad d_{2}+d_{9}>d_{3}+d_{5}, \\
d_{2}+d_{9}>d_{4}, \quad d_{2}+d_{10}>d_{6}, \quad d_{2}+d_{12}>d_{3}+d_{11}, \quad d_{2}+d_{12}>d_{7} \\
d_{3}+d_{5}>d_{4}, \quad d_{3}+d_{10}>d_{7}+d_{9}, \quad d_{3}+d_{10}>d_{8}, \quad d_{3}+d_{11}>d_{7} \\
d_{4}+d_{10}>d_{5}+d_{7}+d_{9}, \quad d_{4}+d_{10}>d_{5}+d_{8}, \quad d_{4}+d_{10}>d_{6}+d_{9} \\
d_{4}+d_{11}>d_{5}+d_{7}, \quad d_{4}+d_{11}>d_{6}, \quad d_{4}+d_{12}>d_{8}, \quad d_{5}+d_{7}>d_{6}, \\
d_{5}+d_{12}>d_{9}+d_{11}, \quad d_{5}+d_{12}>d_{10}, \quad d_{6}+d_{12}>d_{7}+d_{9}+d_{11}, \quad d_{6}+d_{12}>d_{7}+d_{10} \\
d_{6}+d_{12}>d_{8}+d_{11}, \quad d_{7}+d_{9}>d_{8}, \quad d_{9}+d_{11}>d_{10} .
\end{gathered}
$$

For example, $\mathbf{d}=(5,5,1,2,4,1,1,2,6,10,12,20) \in \mathcal{D}_{\underline{w}_{0}}^{q}$. 
Acknowledgements. X.F. is supported by the Alexander von Humboldt Foundation. T.B. and G.F. are partially supported by the DFG priority programme SPP 1388 "Representation Theory".

\section{REFERENCES}

1. T. Backhaus and C. Desczyk, PBW filtration: Feigin-Fourier-Littelmann modules via Hasse diagrams, J. Lie Theory 25(3) (2015), 815-856.

2. T. Backhaus and D. Kus, The PBW filtration and convex polytopes in type B, to appear in Math. Z, arXiv:1504.06522.

3. A. Berenstein and A. Zelevinsky, Tensor product multiplicities, canonical bases and totally positive varieties. Invent. Math. 143(1) (2001), 77-128.

4. G. Cerulli Irelli, X. Fang, E. Feigin, G. Fourier and M. Reineke, Linear degenerations of flag varieties, arXiv:1603.08395.

5. X. Fang and G. Fourier, Marked chain-order polytopes and string polytopes, Eur. J. Comb. 58 (November 2016), 267-282.

6. X. Fang, G. Fourier and P. Littelmann, Essential bases and toric degenerations arising from generating sequences, to appear in Adv. Math, arXiv:1510.02295.

7. X. Fang, G. Fourier and M. Reineke, PBW-Filtration on quantum groups of type $A_{n}$, J. Algebra 449 (2016), 321-345.

8. E. Feigin, G. Fourier and P. Littelmann, PBW filtration and bases for irreducible modules in type $\mathrm{A}_{n}$, Transform. Groups 16(1) (2011), 71-89.

9. E. Feigin, G. Fourier and P. Littelmann, PBW filtration and bases for symplectic Lie algebras, Int. Math. Res. Not. IMRN 24 (2011), 5760-5784.

10. E. Feigin, G. Fourier and P. Littelmann, Favourable modules: Filtrations, polytopes, Newton-Okounkov bodies and flat degenerations, Transform. Groups, 2016, DOI: 10.1007/S00031-016-9389-2.

11. E. Gawrilow and M. Joswig, Polymake: A framework for analyzing convex polytopes. Polytopes - combinatorics and computation (Oberwolfach, 1997), DMV Sem., 29 (Birkhäuser, Basel, 2000), 43-73.

12. A. A. Gornitskii, Essential signatures and canonical bases of irreducible representations of the group $G_{2}$, Math. Notes 97(1) (2015), 30-41.

13. A. A. Gornitskii, Essential signatures and canonical bases of irreducible representations of $D_{4}$, preprint, arXiv:1507.07498.

14. M. Kashiwara, On crystal bases of the q-analogue of universal enveloping algebras, Duke Math. J. 63 (1991), 465-516.

15. V. Kiritchenko, Geometric mitosis, Math. Res. Lett. 23(4) (2016), 1069-1096.

16. V. Kiritchenko, Newton-Okounkov polytopes of flag varieties, to appear in Transform. Groups, DOI:10.1007/s00031-016-9372-y.

17. P. Littelmann, Cones, crystals, and patterns, Transform. Groups 3(2) (1998), 145-179.

18. G. Lusztig, Canonical bases arising from quantized enveloping algebras, J. Amer. Math. Soc. 3(2) (1990), 447-498.

19. G. Lusztig, Introduction to quantum groups. Reprint of the 1994 edition. Modern Birkhäuser Classics (Birkhäuser/Springer, New York, 2010).

20. P. Papi, A characterization of a special ordering in a root system. Proc. Amer. Math. Soc. 120(3) (1994), 661-665. 